Pacific

Journal of

Mathematics

THE EISENSTEIN ELEMENTS OF MODULAR SYMBOLS FOR LEVEL PRODUCT OF TWO DISTINCT ODD PRIMES

DEBARGHA BANERJEE AND SRILAKSHMI KRISHNAMOORTHY 


\title{
THE EISENSTEIN ELEMENTS OF MODULAR SYMBOLS FOR LEVEL PRODUCT OF TWO DISTINCT ODD PRIMES
}

\author{
DEBARGHA BANERJEE AND SRILAKSHMI KRISHNAMOORTHY
}

\begin{abstract}
We explicitly write down the Eisenstein elements inside the space of modular symbols for Eisenstein series with integer coefficients for the congruence subgroups $\Gamma_{0}(p q)$ with $p$ and $q$ distinct odd primes, giving an answer to a question of Merel in these cases. We also compute the winding elements explicitly for these congruence subgroups. Our results are explicit versions of the Manin-Drinfeld theorem.
\end{abstract}

\section{Introduction}

In his landmark paper on Eisenstein ideals, Mazur studied torsion points of elliptic curves over $\mathbb{Q}$ and gave a list of possible torsion subgroups of elliptic curves (see [Mazur 1977, Theorem 8]). Merel [1996b] wrote down modular symbols for the congruence subgroups $\Gamma_{0}(p)$ for any odd prime $p$ that correspond to differential forms of the third kind on the modular curves. He then used these modular symbols to give a uniform upper bound on the torsion points of elliptic curves over any number field in terms of its extension degree [Merel 1996a]. The explicit expressions of winding elements for prime level of [Merel 1996b] were used by Calegari and Emerton [2005] to study the ramifications of Hecke algebras at the Eisenstein primes. Several authors afterwards studied the torsion points of elliptic curves over number fields using modular symbols.

In the present paper, we study elements of relative homology groups of the modular curve $X_{0}(p q)$ that correspond to differential forms of the third kind with $p$ and $q$ distinct odd primes. As a consequence, we give an "effective" proof of the Manin-Drinfeld theorem (Theorem 9) for the special case of the image in $\mathrm{H}_{1}\left(X_{0}(p q), \mathbb{R}\right)$ of the path in $\mathrm{H}_{1}\left(X_{0}(p q), \partial\left(X_{0}(p q)\right), \mathbb{Z}\right)$ joining 0 and $i \infty$. Since the algebraic parts of the special values of the $L$-function are obtained by integrating differential forms on these modular symbols, our explicit expression of the winding elements should be useful for understanding the algebraic parts of the special values at 1 of the $L$-functions of the quotient Jacobian of modular curves for the congruence subgroup $\Gamma_{0}(p q)$ [Agashe 2000].

MSC2010: primary 11F67; secondary $11 \mathrm{~F} 11,11 \mathrm{~F} 20,11 \mathrm{~F} 30$.

Keywords: Eisenstein series, modular symbols, special values of $L$-functions. 
For $N \in\{p, q, p q\}$, consider the basis $E_{N}$ of $E_{2}\left(\Gamma_{0}(p q)\right)$ (Section 4) for which all the Fourier coefficients at $i \infty$ belong to $\mathbb{Z}$. The meromorphic differential forms $E_{N}(z) d z$ are of the third kind on the Riemann surface $X_{0}(p q)$ but of the first kind on the noncompact Riemann surface $Y_{0}(p q)$.

Let $\xi: \mathrm{SL}_{2}(\mathbb{Z}) \rightarrow \mathrm{H}_{1}\left(X_{0}(p q)\right.$, cusps, $\left.\mathbb{Z}\right)$ be the Manin map (Section 3). For any two coprime integers $u$ and $v$ with $v \geq 1$, let $S(u, v) \in \mathbb{Z}$ be the Dedekind sum (see Section 4.1). If $g \in \mathbb{P}^{1}(\mathbb{Z} / p q \mathbb{Z})$ is not of the form $( \pm 1,1),( \pm 1 \pm k x, 1)$ or $(1, \pm 1 \pm k x)$ with $x$ one of the primes $p$ or $q$, then we can write it as $(r-1, r+1)$.

Let $\delta_{r}$ be 1 or 0 depending on whether $r$ is odd or even. For any integer $k$, let $s_{k}=k+\left(\delta_{k}-1\right) p q$ be an odd integer. Choose integers $s, s^{\prime}$ and $l, l^{\prime}$ such that $l\left(s_{k} x+2\right)-2 s p q=1$ and $l^{\prime} s_{k} x-2 s^{\prime} p q / x=1$. Let

$\gamma_{1}^{x, k}=\left(\begin{array}{cc}1+4 s p q & -2 l \\ -4 s\left(s_{k} x+2\right) p q & 1+4 s p q\end{array}\right) \quad$ and $\quad \gamma_{2}^{x, k}=\left(\begin{array}{cc}1+4 s^{\prime} p q / x & -2 l^{\prime} \\ -4 s^{\prime}\left(s_{k}\right) p q & 1+4 s^{\prime} p q / x\end{array}\right)$

be two matrices (see Lemma 28). For $l=1,2$, consider the integers

$$
\begin{aligned}
P_{N}\left(\gamma_{l}^{x, k}\right)=\operatorname{sgn}\left(t\left(\gamma_{l}^{x, k}\right)\right)(2( & \left.S\left(s\left(\gamma_{l}^{x, k}\right),\left|t\left(\gamma_{l}^{x, k}\right)\right| N\right)-S\left(s\left(\gamma_{l}^{x, k}\right),\left|t\left(\gamma_{l}^{x, k}\right)\right|\right)\right) \\
& \left.-S\left(s\left(\gamma_{l}^{x, k}\right), \frac{1}{2}\left|t\left(\gamma_{l}^{x, k}\right)\right| N\right)+S\left(s\left(\gamma_{l}^{x, k}\right), \frac{1}{2}\left|t\left(\gamma_{l}^{x, k}\right)\right|\right)\right)
\end{aligned}
$$

with

$$
s\left(\gamma_{1}^{x, k}\right)=1-4 \operatorname{spq}\left(1+s_{k} x\right), \quad t\left(\gamma_{1}^{x, k}\right)=-2\left(l-2 s\left(s_{k} x+2\right) p q\right)
$$

and

$$
s\left(\gamma_{2}^{x, k}\right)=1-4 s^{\prime} p q\left(s_{k}-\frac{1}{x}\right), \quad t\left(\gamma_{2}^{x, k}\right)=-2\left(l^{\prime}-2 s^{\prime} s_{k} p q\right) .
$$

Define the function $F_{N}: \mathbb{P}^{1}(\mathbb{Z} / p q \mathbb{Z}) \rightarrow \mathbb{Z}$ by

$$
F_{N}(g)= \begin{cases}2(S(r, N)-2 S(r, 2 N)) & \text { if } g=(r-1, r+1), \\ P_{N}\left(\gamma_{1}^{x, k}\right)-P_{N}\left(\gamma_{2}^{x, k}\right) & \text { if } g=(1+k x, 1) \text { or } g=(-1-k x, 1), \\ -P_{N}\left(\gamma_{1}^{x, k}\right)+P_{N}\left(\gamma_{2}^{x, k}\right) & \text { if } g=(1,1+k x) \text { or } g=(1,-1-k x), \\ 0 & \text { if } g=( \pm 1,1) .\end{cases}
$$

Theorem 1. The modular symbol

$$
\mathcal{E}_{E_{N}}=\sum_{g \in \mathbb{P}^{1}(\mathbb{Z} / p q \mathbb{Z})} F_{E_{N}}(g) \xi(g)
$$

in $\mathrm{H}_{1}\left(X_{0}(p q), \partial\left(X_{0}(p q)\right), \mathbb{Z}\right)$ is the Eisenstein element (Section 5) corresponding to the Eisenstein series $E_{N} \in E_{2}\left(\Gamma_{0}(p q)\right)$.

In [Banerjee 2014], a description is given of Eisenstein elements in terms of certain integrals for $M=p^{2}$. In this article, we give an explicit description in 
terms of two matrices $\gamma_{1}^{x, k}$ and $\gamma_{2}^{x, k}$. Let $\bar{B}_{1}: \mathbb{R} \rightarrow \mathbb{R}$ be the periodic first Bernoulli polynomial. For the Eisenstein series $E_{p q}$ (Section 4), we write down the Eisenstein elements more explicitly if $g=(r-1, r+1)$. Replacing $p$ with $p q$ [Merel 1996b, Lemma 4], we write

$$
F_{p q}(r-1, r+1)=\sum_{h=0}^{p q-1} \bar{B}_{1}\left(\frac{h r}{2 p q}\right) .
$$

Recall the concept of the winding elements (Definition 37). We write down the explicit expression of the winding elements for the congruence subgroup $\Gamma_{0}(p q)$.

\section{Corollary 2.}

$$
(1-p q) e_{p q}=\sum_{x \in(\mathbb{Z} / p q \mathbb{Z})^{*}} F_{p q}(1, x)\left\{0, \frac{1}{x}\right\}
$$

Note that if $v=\operatorname{gcd}(p q-1,12)$ and $n=(p q-1) / v$, then a multiple of winding element $n e_{p q}$ belongs to $\mathrm{H}_{1}\left(X_{0}(p q), \mathbb{Z}\right)$. Manin and Drinfeld proved that the modular symbol $\{0, \infty\}$ belongs to $\mathrm{H}_{1}\left(X_{0}(N), \mathbb{Q}\right)$ using the theory of suitable Hecke operators acting on the modular curve $X_{0}(N) / \mathbb{Q}$. In this paper, we follow the approach of Merel [1996b, Proposition 11]. Our explicit expression of winding elements should be useful for understanding the algebraic part of the special values of L-functions (see [Agashe 2000, p. 26]).

Since Hecke operators are defined over $\mathbb{Q}$, there is a possibility that we can find the Eisenstein elements for the congruence subgroups of odd level in a completely different method without using boundary computations. It is tempting to remark that our method should generalize to the congruence subgroup $\Gamma_{0}(N)$ at least if $N$ is squarefree and odd. Unfortunately, generalizing our method is equivalent to having an explicit understanding of boundary homologies of modular curves defined over rationals. For instance, if $N=p q r$ with $p, q, r$ three distinct primes then there are eight cusps. Since there are more cusps in these cases, the computation of boundaries becomes much more tedious. One of the authors wishes to tackle the difficulty using the "level" of the cusps in a future article.

\section{Acknowledgements}

This paper owes its existence to several e-mail communications, encouragements and valuable suggestions from Professor Loïc Merel. We would like to thank IMSc, Chennai, for providing excellent working conditions. Krishnamoorthy would like to thank MPIM, Germany, for the great hospitality during her visit. She was supported by a DST-INSPIRE grant. We wish to thank the anonymous referee for several useful comments that helped us improve the mathematical content of this article. 


\section{Modular symbols}

Let $\mathbb{U} \cup \mathbb{P}^{1}(\mathbb{Q})=\overline{\mathbb{M}}$ and let $\Gamma \subset \mathrm{SL}_{2}(\mathbb{Z})$ be a congruence subgroup. The topological space $X_{\Gamma}(\mathbb{C})=\Gamma \backslash \overline{\mathbb{M}}$ has a natural structure of a smooth compact Riemann surface. Consider the usual projection map $\pi: \overline{\mathbb{M}} \rightarrow X_{\Gamma}(\mathbb{C})$ and recall that it is unramified outside the elliptic points and the set of cusps $\partial\left(X_{\Gamma}\right)$. Both these sets are finite.

3.1. Rational structure of the curve $X_{0}(N)$ defined over $\mathbb{Q}$. There is a smooth projective curve $X_{0}(N)$ defined over $\mathbb{Q}$ for which the space $\Gamma_{0}(N) \backslash \overline{\mathbb{M}}$ is canonically identified with the set of $\mathbb{C}$-points of the projective curve $X_{0}(N)$. We are interested in understanding the $\mathbb{Q}$-structure of the compactified modular curve $X_{0}(N)$.

3.2. Classical modular symbols. Recall the following fundamental theorem.

Theorem 3 [Manin 1972]. For $\alpha \in \overline{\mathbb{M}}$, consider the map $c: \Gamma \rightarrow \mathrm{H}_{1}\left(X_{0}(N), \mathbb{Z}\right)$ defined by

$$
c(g)=\{\alpha, g \alpha\} .
$$

The map $c$ is a surjective group homomorphism which does not depend on the choice of point $\alpha$. The kernel of this homomorphism is generated by

(1) the commutator,

(2) the elliptic elements,

(3) the parabolic elements

of the congruence subgroup $\Gamma$.

In particular, this theorem implies that $\{\alpha, g \alpha\}=0$ for all $\alpha \in \mathbb{P}^{1}(\mathbb{Q})$ and $g \in \Gamma$.

3.3. Manin map. Let $S=\left(\begin{array}{cc}0 & -1 \\ 1 & 0\end{array}\right), T=\left(\begin{array}{ll}1 & 1 \\ 0 & 1\end{array}\right)$ and $R=S T=\left(\begin{array}{cc}0 & -1 \\ 1 & 1\end{array}\right)$. The modular group $\mathrm{SL}_{2}(\mathbb{Z})$ is generated by $S$ and $T$.

Theorem 4 [Manin 1972]. Let

$$
\xi: \mathrm{SL}_{2}(\mathbb{Z}) \rightarrow \mathrm{H}_{1}\left(X_{0}(p q), \partial\left(X_{0}(p q)\right), \mathbb{Z}\right)
$$

be the map that takes a matrix $g \in \mathrm{SL}_{2}(\mathbb{Z})$ to the class in $\mathrm{H}_{1}\left(X_{0}(p q), \partial\left(X_{0}(p q)\right), \mathbb{Z}\right)$

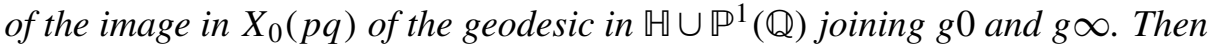

- the map $\xi$ is surjective;

- for all $g \in \Gamma_{0}(p q) \backslash \mathrm{SL}_{2}(\mathbb{Z})$, we have $\xi(g)+\xi(g S)=0$ and $\xi(g)+\xi(g R)+$ $\xi\left(g R^{2}\right)=0$.

We have a short exact sequence

$0 \rightarrow \mathrm{H}_{1}\left(X_{0}(p q), \mathbb{Z}\right) \rightarrow \mathrm{H}_{1}\left(X_{0}(p q), \partial\left(X_{0}(p q)\right), \mathbb{Z}\right) \rightarrow \mathbb{Z}^{\partial\left(X_{0}(p q)\right)} \stackrel{\delta^{\prime}}{\rightarrow} \mathbb{Z} \rightarrow 0$. 
The first map is a canonical injection. The boundary map $\delta^{\prime}$ takes a geodesic, joining the cusps $r$ and $s$ to the formal symbol $[r]-[s]$, and the third map is the sum of the coefficients.

3.4. Relative homology group $\mathrm{H}_{1}\left(X_{0}(p q)-R \cup I, \partial\left(X_{0}(p q)\right)\right.$, $\left.\mathbb{Z}\right)$. Consider the points $i=\sqrt{-1}$ and $\rho=\frac{1}{2}(1+\sqrt{-3})$ on the complex upper half-plane with $v$ the geodesic joining $i$ and $\rho$. These are the elliptic points on the Riemann surface $X_{0}(p q)$. The projection map $\pi$ is unramified outside cusps and elliptic points.

Say $R=\pi\left(\mathrm{SL}_{2}(\mathbb{Z}) \rho\right)$ and let $I=\pi\left(\mathrm{SL}_{2}(\mathbb{Z}) i\right)$ be the image of these two sets in $X_{0}(p q)$. These two sets are disjoint. Consider now the relative homology group $\mathrm{H}_{1}\left(Y_{0}(p q), R \cup I, \mathbb{Z}\right)$. For $g \in \mathrm{SL}_{2}(\mathbb{Z})$, let $[g]_{*}$ be the class of $\pi(g \nu)$ in the relative homology group $\mathrm{H}_{1}\left(Y_{0}(p q), R \cup I, \mathbb{Z}\right)$. Let $\rho^{*}=-\bar{\rho}$ be another point on the boundary of the fundamental domain. The homology groups $\mathrm{H}_{1}\left(Y_{0}(p q), \mathbb{Z}\right)$ are subgroups of $\mathrm{H}_{1}\left(Y_{0}(p q), R \cup I, \mathbb{Z}\right)$. Suppose $z_{0} \in \mathbb{U}$ is such that $\left|z_{0}\right|=1$ and $\frac{-1}{2}<\operatorname{Re}\left(z_{0}\right)<1$. Let $\gamma$ be the union of the geodesics in $\mathbb{U} \cup \mathbb{P}^{1}(\mathbb{Q})$ joining 0 to $z_{0}$ and $z_{0}$ to $i \infty$. For $g \in \Gamma_{0}(p q) \backslash \mathrm{SL}_{2}(\mathbb{Z})$, let $[g]^{*}$ be the class of $\pi(g \gamma)$ in $\mathrm{H}_{1}\left(X_{0}(p q)-R \cup I, \partial\left(X_{0}(p q)\right), \mathbb{Z}\right)$.

We have an intersection pairing

$$
\circ: \mathrm{H}_{1}\left(X_{0}(p q)-R \cup I, \partial\left(X_{0}(p q)\right), \mathbb{Z}\right) \times \mathrm{H}_{1}\left(Y_{0}(p q), R \cup I, \mathbb{Z}\right) \rightarrow \mathbb{Z} .
$$

Recall the following results.

Proposition 5 [Merel 1996b; 1995, Proposition 1]. For $g, h \in \Gamma_{0}(p q) \backslash \mathrm{SL}_{2}(\mathbb{Z})$,

$$
[g]^{*} \circ[h]_{*}= \begin{cases}1 & \text { if } \Gamma_{0}(p q) g=\Gamma_{0}(p q) h, \\ 0 & \text { otherwise. }\end{cases}
$$

Corollary 6 [Merel 1995, Corollary 1]. The homomorphism of groups $\mathbb{Z}^{\Gamma_{0}(p q) \backslash \mathrm{SL}_{2}(\mathbb{Z})}$ $\rightarrow \mathrm{H}_{1}\left(Y_{0}(p q), R \cup I, \mathbb{Z}\right)$ induced by the map

$$
\xi_{0}\left(\sum_{g} \mu_{g} g\right)=\sum_{g} \mu_{g}[g]_{*}
$$

is an isomorphism.

The following important property of the intersection pairing will be used later.

Corollary 7 [Merel 1995, Corollary 3]. For $g \in \Gamma_{0}(p q) \backslash \mathrm{SL}_{2}(\mathbb{Z})$, let $\sum_{h} \mu_{h} h \in$ $\mathbb{Z}^{\Gamma_{0}(p q) \backslash \mathrm{SL}_{2}(\mathbb{Z})}$ be such that $\sum_{h} \mu_{h}[h]_{*}$ is the image of an element of $\mathrm{H}_{1}\left(Y_{0}(p q), \mathbb{Z}\right)$ under the canonical injection. We have

$$
[g]^{*} \circ\left(\sum_{h} \mu_{h}[h]_{*}\right)=\mu_{g} .
$$


We have a short exact sequence

$$
\begin{aligned}
0 \rightarrow \mathrm{H}_{1}\left(X_{0}(p q)\right. & -R \cup I, \mathbb{Z}) \\
& \rightarrow \mathrm{H}_{1}\left(X_{0}(p q)-R \cup I, \partial\left(X_{0}(p q)\right), \mathbb{Z}\right) \rightarrow \mathbb{Z}^{\left\{\partial\left(X_{0}(p q)\right)\right\}} \stackrel{\delta}{\rightarrow} \mathbb{Z} \rightarrow 0 .
\end{aligned}
$$

The boundary map $\delta$ takes a geodesic, joining the cusps $r$ and $s$ to the formal symbol $[r]-[s]$. Note that $\delta^{\prime}(\xi(g))=\delta\left([g]^{*}\right)$ for all $g \in \mathrm{SL}_{2}(\mathbb{Z})$.

Recall that we have a canonical bijection $\Gamma_{0}(p q) \backslash \mathrm{SL}_{2}(\mathbb{Z}) \cong \mathbb{P}^{1}(\mathbb{Z} / p q \mathbb{Z})$ given by $\left(\begin{array}{ll}a & b \\ c & d\end{array}\right) \rightarrow(c, d)$. Say

$$
\alpha_{k}=\left(\begin{array}{cc}
0 & -1 \\
1 & k
\end{array}\right), \quad \beta_{r}=\left(\begin{array}{cc}
-1 & -r \\
p & r p-1
\end{array}\right) \quad \text { and } \quad \gamma_{s}=\left(\begin{array}{cc}
-1 & -s \\
q & s q-1
\end{array}\right) .
$$

We explicitly write down the elements of $\mathbb{P}^{1}(\mathbb{Z} / p q \mathbb{Z})$ as the set

$$
\left\{(1, k),(1, t p),\left(1, t^{\prime} q\right),(p, q),(q, p),(t p, 1),\left(t^{\prime} q, 1\right),(1,0),(0,1)\right\}
$$

with $k \in(\mathbb{Z} / p q \mathbb{Z})^{*}, t \in(\mathbb{Z} / q \mathbb{Z})^{*}, t^{\prime} \in(\mathbb{Z} / p \mathbb{Z})^{*}$. Observe that $(p, q)=(t p, q)=$ $\left(p, t^{\prime} q\right)$ for all $t$ and $t^{\prime}$ coprime to $p q$.

Lemma 8. The set

$$
\Omega=\left\{I, \alpha_{k}, \beta_{r}, \gamma_{s} \mid 0 \leq k \leq p q-1,0 \leq r \leq p-1,0 \leq s \leq q-1\right\}
$$

forms a complete set of coset representatives of $\Gamma_{0}(p q) \backslash \mathrm{SL}_{2}(\mathbb{Z})$.

Proof. The orbits $\Gamma_{0}(p q) \alpha_{k}, \Gamma_{0}(p q) \beta_{l}$ and $\Gamma_{0}(p q) \gamma_{m}$ are disjoint since $a b^{-1}$ does not belong to $\Gamma_{0}(p q)$ for two distinct matrices $a$ and $b$ from the set $\Omega$. There are $1+p q+p+q=\left|\mathbb{P}^{1}(\mathbb{Z} / p q \mathbb{Z})\right|$ coset representatives.

We list different rational numbers of the form $\alpha(0)$ and $\alpha(\infty)$ with $\alpha \in \Omega$ as equivalence classes of cusps as follows:

\begin{tabular}{|c|l|l|}
\hline 0 & $1 / p$ & $1 / q$ \\
\hline$\frac{-l}{l p-1}, \quad(l p-1, q)=1$ & $\frac{-1}{k}, \quad(k, p)>1$ & $\frac{-1}{k}, \quad(k, q)>1$ \\
$\frac{-m}{m q-1},(m q-1, p)=1$ & $\frac{-m}{m q-1},(m q-1, p)>1$ & $\frac{-l}{l p-1},(l p-1, q)>1$ \\
\hline
\end{tabular}

3.5. Manin-Drinfeld theorem. Following [Lang 1995], we briefly recall the statement of the Manin-Drinfeld theorem.

Theorem 9 (Manin-Drinfeld [Drinfeld 1973]). For a congruence subgroup $\Gamma$ and any two cusps $\alpha$ and $\beta$ in $\mathbb{P}^{1}(\mathbb{Q})$, the path

$$
\{\alpha, \beta\} \in \mathrm{H}_{1}\left(X_{\Gamma}, \mathbb{Q}\right) .
$$


This theorem can be reformulated in terms of divisor classes on the Riemann surface.

Theorem 10. Let $a=\sum_{i} m_{i} P_{i}$ be a divisor of degree zero on $X$. Then a is a divisor of a rational function if and only if there exists a cycle $\sigma \in \mathrm{H}_{1}\left(X_{\Gamma}, \mathbb{Z}\right)$ such that

$$
\int_{a} \omega=\sum_{i} m_{i} \int_{P_{0}}^{P_{i}} \omega=\int_{\sigma} \omega
$$

for every $\omega \in \mathrm{H}^{0}\left(X_{\Gamma}, \Omega_{X_{\Gamma}}\right)$.

As a corollary, we notice that $\{x, y\} \in \mathrm{H}_{1}\left(X_{\Gamma}, \mathbb{Q}\right)$ if and only if there is a positive integer $m$ such that $m\left(\pi_{\Gamma}(x)-\pi_{\Gamma}(y)\right)$ is a divisor of a function. In other words, the degree-zero divisors supported on the cusps are of finite order in the divisor class group. Manin and Drinfeld proved it using the extended action of the usual Hecke operators. In particular, it says that $\{0, \infty\} \in \mathrm{H}_{1}\left(X_{\Gamma}, \mathbb{Q}\right)$ although 0 and $\infty$ are two inequivalent cusps of $X_{\Gamma}$. Ogg [1974] constructed a certain modular function $X_{0}(p q)$ whose divisors coincide with degree-zero divisors on the modular curves.

\section{Eisenstein series for $\Gamma_{0}(p q)$ with integer coefficients}

Let $\sigma_{1}(n)$ denote the sum of the positive divisors of $n$. We consider the series

$$
E_{2}^{\prime}(z)=1-24\left(\sum_{n} \sigma_{1}(n) e^{2 \pi i n z}\right) .
$$

Let $\Delta$ be the Ramanujan cusp form of weight 12 . For all $N \in \mathbb{N}$, the function $z \rightarrow \Delta(N z) / \Delta(z)$ is a function on $\mathbb{W}$ invariant under $\Gamma_{0}(N)$. The logarithmic differential of this function is $2 \pi i E_{N}(z) d z$ and $E_{N}$ is a classical holomorphic modular form of weight two for $\Gamma_{0}(N)$ with constant term $N-1$. The differential form $E_{N}(z) d z$ is a differential form of the third kind on $X_{0}(N)$. The periods (Section 4.1) of these differential forms are in $\mathbb{Z}$.

By [Diamond and Shurman 2005, Theorem 4.6.2], the set $\mathbb{E}_{p q}=\left\{E_{p}, E_{q}, E_{p q}\right\}$ is a basis of $E_{2}\left(\Gamma_{0}(p q)\right)$.

Lemma 11. The cusps $\partial\left(X_{0}(p q)\right)$ can be identified with the set $\{0, \infty, 1 / p, 1 / q\}$. Proof. If $a / c$ and $a^{\prime} / c^{\prime}$ are in $\mathbb{P}^{1}(\mathbb{Q})$, then

$$
\Gamma_{0}(p q) \frac{a}{c}=\Gamma_{0}(p q) \frac{a^{\prime}}{c^{\prime}} \Longleftrightarrow\left(\begin{array}{c}
a y \\
c
\end{array}\right) \equiv\left(\begin{array}{c}
a^{\prime}+j c^{\prime} \\
c^{\prime} y
\end{array}\right)(\bmod p q)
$$

for some $j$ and $y$ such that $\operatorname{gcd}(y, p q)=1$ (see [Diamond and Shurman 2005, p. 99]). A small check shows that the orbits $\Gamma_{0}(p q) 0, \Gamma_{0}(p q) \infty, \Gamma_{0}(p q) 1 / p$ and $\Gamma_{0}(p q) 1 / q$ are disjoint. 
Let $\operatorname{Div}^{0}\left(X_{0}(p q), \partial\left(X_{0}(p q)\right), \mathbb{Z}\right)$ be the group of degree-zero divisors supported on cusps. For all cusps $x$, let $e_{\Gamma_{0}(p q)}(x)$ denote the ramification index of $x$ over $\mathrm{SL}_{2}(\mathbb{Z}) \backslash \mathbb{U} \cup \mathbb{P}^{1}(\mathbb{Q})$ and let

$$
r_{\Gamma_{0}(p q)}(x)=e_{\Gamma_{0}(p q)}(x) a_{0}(E[x]) .
$$

By [Stevens 1982, p. 23], there is a canonical isomorphism $\delta: E_{2}\left(\Gamma_{0}(p q)\right) \rightarrow$ $\operatorname{Div}^{0}\left(X_{0}(p q), \partial\left(X_{0}(p q)\right), \mathbb{Z}\right)$ that takes the Eisenstein series $E$ to the divisor

$$
\delta(E)=\sum_{x \in \Gamma_{0}(p q) \backslash \mathbb{P}^{1}(\mathbb{Q})} r_{\Gamma_{0}(p q)}(x)[x] .
$$

Hence, the Eisenstein element is related to the Eisenstein series by the boundary map. In Proposition 34, we prove that the boundary of the Eisenstein element is indeed the boundary of the Eisenstein series. By [Stevens 1985, p. 538], we see that

$$
e_{\Gamma_{0}(p q)}(x)= \begin{cases}q & \text { if } x=1 / p, \\ p & \text { if } x=1 / q, \\ 1 & \text { if } x=\infty \\ p q & \text { if } x=0\end{cases}
$$

Since $\sum_{x \in \partial\left(X_{0}(p q)\right)} e_{\Gamma_{0}(p q)}(x) a_{0}(E[x])=0$, we write the corresponding degree-zero divisor as

$$
\begin{aligned}
\delta(E)=a_{0}(E)(\{\infty\}-\{0\})+q a_{0}\left(E\left[\frac{1}{p}\right]\right)\left(\left\{\frac{1}{p}\right\}\right. & -\{0\}) \\
& +p a_{0}\left(E\left[\frac{1}{q}\right]\right)\left(\left\{\frac{1}{q}\right\}-\{0\}\right) .
\end{aligned}
$$

4.1. Period homomorphisms. We now define period homomorphisms for differential forms of the third kind.

Definition 12 (period homomorphism). For $E_{N} \in \mathbb{E}_{p q}$, the differential forms $E_{N}(z) d z$ are of the third kind on the Riemann surface $X_{0}(p q)$ but of the first kind on the noncompact Riemann surface $Y_{0}(N)$. For any $z_{0} \in \mathbb{U}$ and $\gamma \in \Gamma_{0}(p q)$, let $c(\gamma)$ be the class in $\mathrm{H}_{1}\left(Y_{0}(p q), \mathbb{Z}\right)$ of the image in $Y_{0}(p q)$ of the geodesic in $\mathbb{Z}$ joining $z_{0}$ and $\gamma\left(z_{0}\right)$. That the class is nonzero follows from Theorem 3. This class is independent of the choice of $z_{0} \in \mathbb{M}$. Let $\pi_{E_{N}}(\gamma)=\int_{c(\gamma)} E_{N}(z) d z$. The map $\pi_{E_{N}}: \Gamma_{0}(p q) \rightarrow \mathbb{Z}$ is the "period" homomorphism of $E_{N}$.

Let $\bar{B}_{1}(x)$ be the first Bernoulli polynomial of period one defined by

$$
\bar{B}_{1}(0)=0, \quad \bar{B}_{1}(x)=x-\frac{1}{2}
$$


if $x \in(0,1)$. For any two integers $u$ and $v$ with $v \geq 1$, we define the Dedekind sum

$$
S(u, v)=\sum_{t=1}^{v-1} \bar{B}_{1}\left(\frac{t u}{v}\right) \bar{B}_{1}\left(\frac{u}{v}\right) .
$$

Recall some well-known properties of the period mapping $\pi_{E_{N}}$ (see [Mazur 1979, p. 10; Merel 1996b, p. 14]) for the Eisenstein series $E_{N} \in \mathbb{E}_{p q}$.

Proposition 13. Let $\gamma=\left(\begin{array}{ll}a & b \\ c & d\end{array}\right)$ be an element of $\Gamma_{0}(p q)$. Then

(1) $\pi_{E_{N}}$ is a homomorphism $\Gamma_{0}(p q) \rightarrow \mathbb{Z}$;

(2) the image of $\pi_{E_{N}}$ lies in $\mu \mathbb{Z}$, where $\mu=\operatorname{gcd}(N-1,12)$;

(3) $\pi_{E_{N}}(\gamma)= \begin{cases}\frac{a+d}{c}(N-1)+12 \operatorname{sgn}(c)\left(S(d,|c|)-S\left(d, \frac{|c|}{N}\right)\right) & \text { if } c \neq 0, \\ \frac{b}{d}(N-1) & \text { if } c=0 ;\end{cases}$

(4) $\pi_{E_{N}}(\gamma)=\pi_{E_{N}}\left(\left(\begin{array}{cc}d & c / N \\ N b & a\end{array}\right)\right)$.

\section{Eisenstein elements}

Following [Merel 1996b] and [Merel 1993], we recall the concept of Eisenstein elements of the space of modular symbols. For any natural number $M>4$, the congruence subgroup $\Gamma_{0}(M)$ is the subgroup of $\mathrm{SL}_{2}(\mathbb{Z})$ consisting of all matrices $\left(\begin{array}{ll}a & b \\ c & d\end{array}\right)$ such that $M \mid c$. The congruence subgroup $\Gamma_{0}(M)$ acts on the upper half-plane $\mathbb{W}$ in the usual way. The quotient space $\Gamma_{0}(M) \backslash \mathbb{M}$ is denoted by $Y_{0}(M)$. A priori, these are all Riemann surfaces and hence algebraic curves defined over $\mathbb{C}$. There are models of these algebraic curves defined over $\mathbb{Q}$ and they parametrize elliptic curves with cyclic subgroups of order $M$. Let $X_{0}(M)$ be the compactification of the Riemann surface $Y_{0}(M)$ obtained by adjoining the set of cusps $\partial\left(X_{0}(M)\right)=\Gamma_{0}(M) \backslash \mathbb{P}^{1}(\mathbb{Q})$.

Definition 14 (Eisenstein elements). Let $\pi_{E_{N}}: \mathrm{H}_{1}\left(Y_{0}(p q), \mathbb{Z}\right) \rightarrow \mathbb{Z}$ be the period homomorphism of $E_{N}$ (Section 4.1). The intersection pairing $\circ$ [Merel 1993] induces a perfect, bilinear pairing

$$
\mathrm{H}_{1}\left(X_{0}(p q), \partial\left(X_{0}(p q)\right), \mathbb{Z}\right) \times \mathrm{H}_{1}\left(Y_{0}(p q), \mathbb{Z}\right) \rightarrow \mathbb{Z} .
$$

Since $\circ$ is a nondegenerate bilinear pairing, there is a unique element $\mathcal{E}_{E_{N}} \in$ $\mathrm{H}_{1}\left(X_{0}(p q), \partial\left(X_{0}(p q)\right), \mathbb{Z}\right)$ such that $\mathcal{E}_{E_{N}} \circ c=\pi_{E_{N}}(c)$. The modular symbol $\mathcal{E}_{E_{N}}$ is the Eisenstein element corresponding to the Eisenstein series $E_{N}$.

We intersect with the congruence subgroup $\Gamma(2)$ to ensure that the Manin maps become bijective (rather than only surjective), compute the Eisenstein elements for these modular curves, calculate the boundaries and show that these boundaries 
coincide with the original Eisenstein elements. In the case of $\Gamma_{0}\left(p^{2}\right)$, although it is difficult to find the Fourier expansion of modular forms at different cusps, fortunately for all $g \in \Gamma_{0}(p)$ the matrices $g\left(\begin{array}{ll}1 & 1 \\ 0 & 1\end{array}\right) g^{-1}$ belong to $\Gamma_{0}\left(p^{2}\right)$, and hence it is easier to tackle the explicit coset representatives. Unfortunately, for $N=p q$ or $N=p^{3}$ this is no longer true.

To get around this problem for the congruence subgroup $\Gamma_{0}(p q)$ with $p$ and $q$ distinct primes, we use the relative homology groups $\mathrm{H}_{1}\left(X_{0}(p q), R \cup I, \mathbb{Z}\right)$. For these relative homology groups, the associated Manin maps are bijective and the push forward of the Eisenstein elements inside the original modular curves turns out to have the same boundary as the original Eisenstein elements. We consider three different homology groups in this paper. In particular, the study of the relative homology group $\mathrm{H}_{1}\left(X_{0}(N), R \cup I, \mathbb{Z}\right)$ to determine the Eisenstein element is a new idea. That these relative homology groups should be useful in the study of modular symbols was discovered by Merel.

Definition 15 (almost Eisenstein elements). For $N \in\{p, q, p q\}$, the differential form $E_{N}(z) d z$ is of the first kind on the Riemann surface $Y_{0}(p q)$. Since $\circ$ is a nondegenerate bilinear pairing, there is a unique element

$$
\mathcal{E}_{E_{N}}^{\prime} \in \mathrm{H}_{1}\left(X_{0}(p q)-R \cup I, \partial\left(X_{0}(p q)\right), \mathbb{Z}\right)
$$

such that $\mathcal{E}_{E_{N}}^{\prime} \circ c=\pi_{E_{N}}(c)$ for all $c \in \mathrm{H}_{1}\left(Y_{0}(p q), R \cup I, \mathbb{Z}\right)$. We call $\mathcal{E}_{E_{N}}^{\prime}$ the almost Eisenstein element corresponding to the Eisenstein series $E_{N}$.

\section{Even Eisenstein elements}

\subsection{Simply connected Riemann surface of genus zero with three marked points.} Recall that there is only one simply connected (genus zero) compact Riemann surface up to conformal bijections: namely, the Riemann sphere or the projective complex plane $\mathbb{P}^{1}(\mathbb{C})$. A theorem of Belyi states that every compact, connected, nonsingular algebraic curve $X$ has a model defined over $\overline{\mathbb{Q}}$ if and only if it admits a map to $\mathbb{P}^{1}(\mathbb{C})$ branched over three points.

Consider the subgroup $\Gamma(2)$ of $\mathrm{SL}_{2}(\mathbb{Z})$ consisting of all matrices which are the identity modulo the reduction map modulo 2 . The Riemann surface $\Gamma(2) \bmod \overline{\mathbb{T}}$ is a Riemann surface of genus zero, denoted by $X(2)$. Hence, it can be identified with $\mathbb{P}^{1}(\mathbb{C})$.

The subgroup $\Gamma(2)$ has three cusps $\Gamma(2) 0, \Gamma(2) 1$ and $\Gamma(2) \infty$. Hence, $\Gamma(2) \backslash \overline{\mathbb{M}}$ becomes the simply connected Riemann surface $\mathbb{P}^{1}(\mathbb{C})$ with the three marked points $\Gamma(2) 0, \Gamma(2) 1$ and $\Gamma(2) \infty$ given by the respective cusps. The modular curve $X_{0}(p q)$ has no obvious morphism to $X(2)$. So we consider the modular curve $X_{\Gamma}$ (Section 6.2). There are two obvious maps $\pi, \pi^{\prime}$ from $X_{\Gamma}$ to the compact Riemann surface $X_{0}(p q)$. 
6.2. Modular curves with bijective Manin maps. For the congruence subgroup $\Gamma=\Gamma_{0}(p q) \cap \Gamma(2)$, consider the compactified modular curve $X_{\Gamma}=\Gamma \backslash \mathbb{M} \cup \mathbb{P}^{1}(\mathbb{Q})$ and let $\pi_{\Gamma}: \mathbb{U} \cup \mathbb{P}^{1}(\mathbb{Q}) \rightarrow X_{\Gamma}$ be the canonical surjection.

Let $\pi_{0}: \Gamma \backslash \mathbb{H} \cup \mathbb{P}^{1}(\mathbb{Q}) \rightarrow \Gamma(2) \backslash \mathbb{U} \cup \mathbb{P}^{1}(\mathbb{Q})$ be the map $\pi_{0}(\Gamma z)=\Gamma(2) z$. The compact Riemann surface $X(2)$ contains three cusps $\Gamma(2) 1, \Gamma(2) 0$ and $\Gamma(2) \infty$. Let $P_{-}=\pi_{0}^{-1}(\Gamma(2) 1)$ and let $P_{+}$be the union of two sets $\pi_{0}^{-1}(\Gamma(2) 0)$ and $\pi_{0}^{-1}(\Gamma(2) \infty)$. Consider now the Riemann surface $X_{\Gamma}$ with boundary $P_{+}$and $P_{-}$.

Let $\delta_{r}$ be 1 or 0 depending on whether $r$ is odd or even. For any integer $k$, let $s_{k}=k+\left(\delta_{k}-1\right) p q$ be an odd integer. Let $l$ and $m$ be two unique integers such that $l q+m p \equiv 1(\bmod p q)$ with $1 \leq l \leq p-1$ and $1 \leq m \leq q-1$. The matrices

$$
\begin{aligned}
\alpha_{p q}^{\prime} & =\left(\begin{array}{cc}
p q & p q-1 \\
p q+1 & p q
\end{array}\right), \\
\alpha_{k}^{\prime} & =\left(\begin{array}{cc}
s_{k}(p q)^{2} & s_{k} p q-1 \\
s_{k} p q+1 & s_{k}
\end{array}\right), \\
\beta_{r}^{\prime} & =\left(\begin{array}{cc}
-1 & -\left(r+\delta_{r} q\right) \\
p+p q & -1+\left(r+\delta_{r} q\right)(p+p q)
\end{array}\right), \\
\gamma_{s}^{\prime} & =\left(\begin{array}{cc}
-1 & -\left(s+\delta_{s} p q\right) \\
q+p q & -1+\left(s+\delta_{s} p q\right)(q+p q)
\end{array}\right)
\end{aligned}
$$

are useful for calculating the boundaries of the Eisenstein elements.

Lemma 16. The set

$$
\Delta=\left\{I, \alpha_{k}^{\prime}, \beta_{r}^{\prime}, \gamma_{s}^{\prime} \mid 0 \leq k \leq p q-1,0 \leq r \leq q-1,0 \leq s \leq p-1\right\} \subset \Gamma(2)
$$

forms an explicit set of coset representatives of $\mathbb{P}^{1}(\mathbb{Z} / p q \mathbb{Z})$.

Proof. An easy check shows that the orbits $\Gamma_{0}(p q) \alpha_{k}^{\prime}, \Gamma_{0}(p q) \beta_{r}^{\prime}$ and $\Gamma_{0}(p q) \gamma_{s}^{\prime}$ are disjoint. Since $\left|\mathbb{P}^{1}(\mathbb{Z} / p q \mathbb{Z})\right|=p q+p+q+1$, the result follows.

The coset representatives in the above lemma are chosen such that $\Gamma_{0}(p q) \beta_{r}=$ $\Gamma_{0}(p q) \beta_{r}^{\prime}$ and $\Gamma_{0}(p q) \gamma_{s}=\Gamma_{0}(p q) \gamma_{s}^{\prime}$.

Lemma 17. $\Gamma \backslash \Gamma(2)$ is isomorphic to $\mathbb{P}^{1}(\mathbb{Z} / p q \mathbb{Z})$.

Proof. The explicit coset representatives of Lemma 16 produce the canonical bijection.

We study the relative homology groups $\mathrm{H}_{1}\left(X_{\Gamma}-P_{-}, P_{+}, \mathbb{Z}\right)$ and $\mathrm{H}_{1}\left(X_{\Gamma}-P_{+}\right.$, $\left.P_{-}, \mathbb{Z}\right)$. The intersection pairing is a nondegenerate bilinear pairing $\circ: \mathrm{H}_{1}\left(X_{\Gamma}-P_{+}\right.$, $\left.P_{-}, \mathbb{Z}\right) \times \mathrm{H}_{1}\left(X_{\Gamma}-P_{-}, P_{+}, \mathbb{Z}\right) \rightarrow \mathbb{Z}$. For $g \in \Gamma \backslash \Gamma(2)$, let $[g]^{0}$ (respectively $[g]_{0}$ ) be

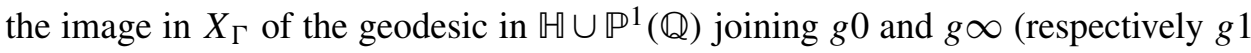
and $g(-1)$ ). Recall the following fundamental theorems. 
Theorem 18 [Merel 1996b]. Let

$$
\xi_{0}: \mathbb{Z}^{\Gamma \backslash \Gamma(2)} \rightarrow \mathrm{H}_{1}\left(X_{\Gamma}-P_{+}, P_{-}, \mathbb{Z}\right)
$$

be the map which takes $g \in \Gamma \backslash \Gamma(2)$ to the element $[g]_{0}$ and

$$
\xi^{0}: \mathbb{Z}^{\Gamma \backslash \Gamma(2)} \rightarrow \mathrm{H}_{1}\left(X_{\Gamma}-P_{-}, P_{+}, \mathbb{Z}\right)
$$

be the map which takes $g \in \Gamma \backslash \Gamma(2)$ to the element $[g]^{0}$. The homomorphisms $\xi_{0}$ and $\xi^{0}$ are isomorphisms.

Theorem 19 [Merel 1996b]. For $g, g^{\prime} \in \Gamma(2)$, we have

$$
[g]_{0} \circ\left[g^{\prime}\right]^{0}= \begin{cases}1 & \text { if } \Gamma g=\Gamma g^{\prime}, \\ 0 & \text { otherwise. }\end{cases}
$$

The following two lemmas about the set $P_{-}$are true for the congruence subgroup $\Gamma_{0}(N)$ with $N$ odd.

Lemma 20. We can explicitly write the elements of the set $P_{-}$in the form $\Gamma x / y$ with $x$ and $y$ both odd.

Proof. Suppose that some element of $P_{-}$is of the form $\Gamma x / y$ with $x$ and $y$ coprime and $y$ even. Consider the corresponding element in the marked simply connected Riemann surface $X(2)$. The cusp $\Gamma(2) x / y$ is an element such that $y$ is even and $p$ is odd $(\operatorname{gcd}(x, y)=1)$. First, choose $p^{\prime}, q^{\prime}$ such that $x q^{\prime}-y p^{\prime}=1$ and hence

$$
D=\left(\begin{array}{ll}
x & p^{\prime} \\
y & q^{\prime}
\end{array}\right) \in \mathrm{SL}_{2}(\mathbb{Z}) \text {. }
$$

Clearly, $q^{\prime}$ is odd since $y$ is even. If $p^{\prime}$ is odd then replace the matrix $D$ with $D T^{-1}$ to produce a matrix in $\Gamma(2)$ that takes $i \infty$ to $x / y$. This contradicts $\Gamma x / y \in P_{-}$.

If $x$ is even then the projection of $\Gamma x / y$ produces an element of $\Gamma(2) 0$. So $x$ is necessarily odd.

The following lemma is deeply influenced by important results of Manin [1972, Proposition 2.2] and Cremona [1997, Proposition 2.2.3].

Corollary 21. We can explicitly write the set $P_{-}$as $\{\Gamma 1, \Gamma 1 /(p q), \Gamma 1 / p, \Gamma 1 / q\}$.

Proof. Since $P_{-}=\pi_{0}^{-1}(\Gamma(2) 1)$, we can write every element of the set $P_{-}$as $\Gamma \theta 1$ for some $\theta \in \Delta$ (Lemma 16). Let $\delta \in\{1, p, q, p q\}$. Then every element of $P_{-}$ can be written as $\Gamma u /(v \delta)$ with $\operatorname{gcd}(u, v \delta)=1$ and $\operatorname{gcd}(v \delta, p q / \delta)=1$. Choose an odd integer $m$ and an even integer $l$ such that $l u-m v \delta=1$. Matrix multiplication shows that

$$
\left(\begin{array}{cc}
1 & 0 \\
\delta-1 & 1
\end{array}\right)\left(\begin{array}{cc}
1+c & -c \\
c & 1-c
\end{array}\right)=\frac{1}{\delta}
$$


and

$$
\left(\begin{array}{cc}
-m & u+m \\
-l & l+v \delta
\end{array}\right)=\frac{u}{v \delta}
$$

and hence

$$
A=\left(\begin{array}{cc}
1 & 0 \\
\delta-1 & 1
\end{array}\right)\left(\begin{array}{cc}
1+c & -c \\
c & 1-c
\end{array}\right)\left(\begin{array}{cc}
l+v \delta & -m-u \\
l & -m
\end{array}\right)
$$

is a matrix such that $A(u /(v \delta))=1 / \delta$. The matrix $A$ belongs to $\Gamma$ if and only if $c v \delta \equiv l^{\prime}(\bmod p q / \delta)$. Since $v \delta$ is coprime to $p q / \delta$, there is always such a $c$. Hence, the set $P_{-}$consists of the four elements given in the statement of the corollary.

Let $\pi, \pi^{\prime}: \Gamma \backslash \overline{\mathbb{M}} \rightarrow \Gamma_{0}(p q) \backslash \overline{\mathbb{M}}$ be the maps $\pi(\Gamma z)=\Gamma_{0}(p q) z$ and $\pi^{\prime}(\Gamma z)=$ $\Gamma_{0}(p q) \frac{1}{2}(z+1)$ respectively. Consider the matrix $h=\left(\begin{array}{ll}1 & 1 \\ 0 & 2\end{array}\right)$. The morphism $\pi^{\prime}$ is well defined since the matrix $h \gamma h^{-1}$ belongs to $\Gamma_{0}(p q)$ for all $\gamma \in \Gamma$. The morphisms $\pi, \pi^{\prime}$ together induce a map

$$
\kappa: \mathbb{C}\left(X_{\Gamma}\right) \rightarrow \mathbb{C}\left(X_{0}(p q)\right)
$$

between the function fields of the Riemann surfaces $X_{\Gamma}$ given by $\kappa(f(z))=$ $f(\pi(\Gamma z))^{2} / f\left(\pi^{\prime}(\Gamma z)\right)$. Recall the description of the coordinate chart around a cusp $\Gamma x$ [Miyake 1976] of the Riemann surface $X_{\Gamma}$.

Definition 22. For a cusp $y$ of the congruence subgroup $\Gamma$, let $\Gamma_{y}$ be the subgroup of $\Gamma$ fixing $y$. Let $t \in \mathrm{SL}_{2}(\mathbb{R})$ be such that $t(y)=i \infty$ and let $m$ be the smallest natural number such that $t \Gamma_{y} t^{-1}$ is generated by $\left(\begin{array}{ll}1 & m \\ 0 & 1\end{array}\right)$. For the modular curve $X_{\Gamma}$, the local coordinate around the point $\Gamma y$ is given by $z \rightarrow e^{2 \pi i t(z) / m}$.

Example 23. Let $y=1 / \delta$ with $\delta$ one of the primes $p$ or $q$. Then $h(y)=u / \delta$ with $(u, p q)=1$. Choose integers $u^{\prime}, \delta^{\prime}$ with $\delta^{\prime}$ even such that $u \delta^{\prime}-u^{\prime} \delta=1$; hence $\rho_{h(y)}=\left(\begin{array}{cc}\delta^{\prime} & u^{\prime} \\ -\delta & u\end{array}\right)$ is such that $\rho_{h(y)}(h(y))=i \infty$. We can choose such a $\delta^{\prime} \in \mathbb{Z}$ since $\delta$ is odd.

Matrix multiplication shows that

$$
\rho_{h(y)} T^{e} \rho_{h(y)}{ }^{-1}=\left(\begin{array}{cc}
1+e \delta \delta^{\prime} & e\left(\delta^{\prime}\right)^{2} \\
-e \delta^{2} & 1-e \delta \delta^{\prime}
\end{array}\right) .
$$

Hence, the smallest possible $e$ to ensure $t T^{e} t^{-1} \subset \Gamma_{0}(p q)$ is $p q / \delta$.

Example 24. Since $\operatorname{det}\left(\rho_{h(y)} \circ h\right)=2$,

$$
t=\left(\begin{array}{cc}
\frac{1}{2} l & 0 \\
0 & 1
\end{array}\right) \rho_{h(y)} \circ h \in \mathrm{SL}_{2}(\mathbb{R})
$$

and $t(y)=i \infty$. A calculation shows that

$$
t T^{e} t^{-1}=\left(\begin{array}{cc}
1+\frac{1}{2} e \delta \delta^{\prime} & \frac{1}{4} e \delta^{\prime 2} \\
-e \delta^{2} & 1-\frac{1}{2} e \delta \delta^{\prime}
\end{array}\right)
$$


Hence, the smallest possible $e$ to ensure $t T^{e} t^{-1} \subset \Gamma$ is $e=2 p q / \delta$.

We use the following lemma to construct differential forms of the first kind on the ambient Riemann surface $X_{\Gamma}-P_{+}$.

Lemma 25. Let $f: X_{0}(p q) \rightarrow \mathbb{C}$ be a rational function. The divisors of $\kappa(f)$ are supported on $P_{+}$.

Proof. Suppose $f$ is a meromorphic function on the Riemann surface $X_{0}(p q)$. Then $f$ is given by $g / h$ with $g$ and $h$ holomorphic functions on $X_{0}(p q)$. Every element of $P_{-}$is of the form $\Gamma 1 / \delta$ with $\delta \mid N$. By [Miranda 1995, Proposition 4.1], every holomorphic map on a Riemann surface locally looks like $z \rightarrow z^{n}$.

Consider the morphism $\pi^{\prime}$ and the point on the modular curve $\Gamma 1 / \delta$. The local coordinates around the points $\Gamma_{0}(p q) 0, \Gamma_{0}(p q) \infty$ and $\Gamma_{0}(p q) 1 / p$ are given by $q_{0}(z)=e^{2 \pi i /(-p q z)}, q_{\infty}(z)=e^{2 \pi i z}$ and $q_{1 / q}(z)=e^{2 \pi i z /(p(-q z+1))}$ respectively. In the modular curve $X_{\Gamma}$, the local coordinates around the points of $P_{-}$are given by

$$
\begin{aligned}
q_{1}(z) & =e^{2 \pi i /(2 p q(-z+1))}, \\
q_{1 /(p q)}(z) & =e^{2 \pi i z /(2(-p q z+1))}, \\
q_{1 / p}(z) & =e^{2 \pi i z /(2 q(-p z+1))}, \\
q_{1 / q}(z) & =e^{2 \pi i z /(2 p(-q z+1))} .
\end{aligned}
$$

Now around the points $\Gamma 1$ and $\Gamma 1 /(p q)$ we have the equalities $q_{0} \circ \pi=q_{1}^{2}$, $q_{0} \circ \pi^{\prime}=q_{1}^{4}$ and $q_{1 /(p q)} \circ \pi=q_{1 /(p q)}^{2}, q_{1 /(p q)} \circ \pi^{\prime}=q_{1 /(p q)}^{4}$.

Let $y=1 / \delta$ with $\delta$ one of the primes $p$ or $q$. The local coordinate chart around the point $\Gamma 1 / \delta$ is $z \rightarrow e^{2 \pi i \rho_{h(x)} \circ h(z) /(4 e)}$. The map $\pi^{\prime}$ takes it to $e^{2 \pi i 2 \rho_{h(x)}(h(z)) / e}$. For this coordinate chart the map $\pi^{\prime}$ is given by $z \rightarrow z^{4}$.

We now consider the map $\pi$ and a matrix $t=\left(\begin{array}{cc}1 & 0 \\ -\delta & 1\end{array}\right)$ such that $t(y)=i \infty$ and $e=p q / \delta$. The local coordinate around the point $\Gamma 1 / \delta$ is $z \rightarrow e^{2 \pi i t(z) /(2 e)}$ and the map $\pi$ takes it to $e^{2 \pi i t(z) / e}$. In this coordinate chart, the map $\pi$ is given by $z \rightarrow z^{2}$. Hence, the function $(f \circ \pi)^{2} /\left(f \circ \pi^{\prime}\right)$ has no zero or pole on $P_{-}$.

Definition 26 (even Eisenstein elements). For $E_{N} \in \mathbb{E}_{p q}$, let $\lambda_{E_{N}}: X_{0}(p q) \rightarrow \mathbb{C}$ be the rational function whose logarithmic differential is $2 \pi i E_{N}(z) d z=2 \pi i \omega_{E_{N}}$. Consider the rational function $\lambda_{E_{N}, 2}=\left(\lambda_{E_{N}} \circ \pi\right)^{2} /\left(\lambda_{E_{N}} \circ \pi^{\prime}\right)$ on $X_{\Gamma}$. By Lemma 25 , this function has no zeros and poles in $P_{-}$. Let $\kappa^{*}\left(\omega_{E_{N}}\right)$ be the logarithmic differential of the function. Let $\varphi_{E_{N}}(c)=\int_{c} \kappa^{*}\left(\omega_{E_{N}}\right)$ be the corresponding period homomorphism $\mathrm{H}_{1}\left(X_{\Gamma}-P_{+}, P_{-}, \mathbb{Z}\right) \rightarrow \mathbb{Z}$.

By the nondegeneracy of the intersection pairing, there is a unique element $\mathcal{E}_{E_{N}}^{0} \in \mathrm{H}_{1}\left(X_{\Gamma}-P_{-}, P_{+}, \mathbb{Z}\right)$ such that $\mathcal{E}_{E_{N}}^{0} \circ c=\varphi_{E_{N}}(c)$ for all $c \in \mathrm{H}_{1}\left(X_{\Gamma}-P_{+}, P_{-}, \mathbb{Z}\right)$. The modular symbol $\mathcal{E}_{E_{N}}^{0}$ is the even Eisenstein element corresponding to the Eisenstein series $E_{N}$. 
For $E_{N} \in \mathbb{E}_{p q}$, define a function $F_{E_{N}}: \mathbb{P}^{1}(\mathbb{Z} / p q \mathbb{Z}) \rightarrow \mathbb{Z}$ by

$$
F_{E_{N}}(g)=\varphi_{E_{N}}\left(\xi_{0}(g)\right)=\int_{g(1)}^{g(-1)}\left(2 E_{N}(z)-E_{N}\left(\frac{1}{2}(z+1)\right)\right) d z
$$

Remark 27. It is easy to see that for any $\gamma=\left(\begin{array}{ll}a & b \\ c & d\end{array}\right) \in \Gamma(2)$,

$$
h \gamma h^{-1}=\left(\begin{array}{cc}
a+c & \frac{1}{2}(b+d-a-c) \\
2 c & d-c
\end{array}\right) \in \mathrm{SL}_{2}(\mathbb{Z}) .
$$

For any matrix $\gamma \in \Gamma$, consider the rational numbers

$$
\begin{aligned}
P_{N}(\gamma) & =\frac{1}{12}\left(2 \pi_{E_{N}}(\gamma)-\pi_{E_{N}}\left(h \gamma h^{-1}\right)\right), \\
t(\gamma) & =b+d-a-c, \\
s(\gamma) & =a+c .
\end{aligned}
$$

Lemma 28. For $\gamma=\left(\begin{array}{ll}a & b \\ c & d\end{array}\right) \in \Gamma$ with $c \neq 0$,

$$
\begin{aligned}
P_{N}(\gamma)=\operatorname{sgn}(t(\gamma))(2(S(s(\gamma),|t(\gamma)| p q) & -S(s(\gamma),|t(\gamma)|)) \\
& \left.-S\left(s(\gamma),\left|\frac{1}{2} t(\gamma)\right| p q\right)+S\left(s(\gamma), \frac{1}{2}|t(\gamma)|\right)\right) .
\end{aligned}
$$

In particular, $P_{N}(\gamma) \in \mathbb{Z}$ for all $\gamma \in \Gamma$.

Proof. Recall the properties of period homomorphism (see Proposition 13). We calculate the corresponding periods:

$$
\begin{aligned}
\pi_{E_{N}}(\gamma) & =\pi_{E}\left(T \gamma T^{-1}\right) \\
& =\pi_{E_{N}}\left(\left(\begin{array}{cc}
a+c & -(a+c)+b+d \\
c & -c+d
\end{array}\right)\right) \\
& =\pi_{E_{N}}\left(\left(\begin{array}{cc}
a+c & -(a+c)+b+d \\
c & -c+d
\end{array}\right)\right) \\
& =\pi_{E_{N}}\left(\left(\begin{array}{cc}
d-c & c / N \\
t(\gamma) N & a+c
\end{array}\right)\right) .
\end{aligned}
$$

By Proposition 13, we have

$$
\pi_{E_{N}}(\gamma)=\frac{a+d}{t(\gamma) N}(N-1)+12 \operatorname{sgn}(t(\gamma))(S(s(\gamma),|t(\gamma)| N)-S(s(\gamma),|t(\gamma)|)) .
$$


Similarly,

$$
\begin{aligned}
& \pi_{E_{N}}\left(h \gamma h^{-1}\right) \\
& \quad=\pi_{E_{N}}\left(\left(\begin{array}{cc}
a+c & \frac{1}{2}(b+d-a-c) \\
2 c & d-c
\end{array}\right)\right) \\
& \quad=\pi_{E_{N}}\left(\left(\begin{array}{cc}
d-c & 2 c / N \\
\frac{1}{2} t(\gamma) N^{2} & a+c
\end{array}\right)\right) \\
& \quad=\frac{2(a+d)}{t(\gamma) N}(N-1)+12 \operatorname{sgn}(t(\gamma))\left(S\left(s(\gamma), \frac{1}{2}|t(\gamma)| N\right)-S\left(s(\gamma), \frac{1}{2}|t(\gamma)|\right)\right) .
\end{aligned}
$$

Hence, we deduce the formula given in the lemma statement. From the formula, we see that $P_{N}(\gamma) \in \mathbb{Z}$ for all $\gamma \in \Gamma$.

Let $x$ be one of the primes $p$ or $q$. Choose integers $s, s^{\prime}$ and $l, l^{\prime}$ such that $l\left(s_{k} x+2\right)-2 s p q=1$ and $l^{\prime} s_{k} x-2 s^{\prime} p q / x=1$. Let

$\gamma_{1}^{x, k}=\left(\begin{array}{cc}1+4 s p q & -2 l \\ -4 s\left(s_{k} x+2\right) p q & 1+4 s p q\end{array}\right) \quad$ and $\quad \gamma_{2}^{x, k}=\left(\begin{array}{cc}1+4 s^{\prime} p q / x & -2 l^{\prime} \\ -4 s^{\prime}\left(s_{k}\right) p q & 1+4 s^{\prime} p q / x\end{array}\right)$

be two matrices in $\Gamma$. Since the integers $l$ and $l^{\prime}$ are necessarily odd, we have $\gamma_{1}^{x, k}\left(1 /\left(s_{k} x+2\right)\right)=-1 /\left(s_{k} x+2\right)$ and $\gamma_{2}^{x, k}\left(1 /\left(s_{k} x\right)\right)=-1 /\left(s_{k} x\right)$.

Using the formula of Lemma 28, we deduce that

$$
s\left(\gamma_{1}^{x, k}\right)=1-4 \operatorname{spq}\left(1+s_{k} x\right), \quad t\left(\gamma_{1}^{x, k}\right)=-2\left(l-2 s\left(s_{k} x+2\right) p q\right)
$$

and

$$
s\left(\gamma_{2}^{x, k}\right)=1-4 s^{\prime} p q\left(s_{k}-\frac{1}{x}\right), \quad t\left(\gamma_{2}^{x, k}\right)=-2\left(l^{\prime}-2 s^{\prime} s_{k} p q\right) .
$$

We can now calculate $P_{N}\left(\gamma_{1}^{x, k}\right)$ and $P_{N}\left(\gamma_{2}^{x, k}\right)$ using Lemma 28 .

\section{Proposition 29.}

$$
F_{E_{N}}(g)= \begin{cases}12(S(r, N)-2 S(r, 2 N)) & \text { if } g=(r-1, r+1), \\ 6\left(P_{N}\left(\gamma_{1}^{x, k}\right)-P_{N}\left(\gamma_{2}^{x, k}\right)\right) & \text { if } g=(1+k x, 1) \text { or } g=(-1-k x, 1), \\ -6\left(P_{N}\left(\gamma_{1}^{x, k}\right)-P_{N}\left(\gamma_{2}^{x, k}\right)\right) & \text { if } g=(1,-1-k x) \text { or } g=(1,1+k x), \\ 0 & \text { if } g=( \pm 1,1) .\end{cases}
$$

Proof. If $g=(r-1, r+1)$ and $E_{N} \in \mathbb{E}_{p q}$, we get [Merel 1996b, p. 18]

$$
F_{E_{N}}(g)=\varphi_{E_{N}}\left(\xi_{0}(g)\right)=12(S(r, N)-2 S(r, 2 N)) .
$$

We now find the value of the integrals in the remaining cases. The differential form $k^{*}\left(\omega_{E_{N}}\right)$ is of the first kind on the Riemann surface $X_{\Gamma}-P_{+}$. We also note that if $g=( \pm 1,1),( \pm 1 \pm k x, 1)$ or $(1, \pm 1 \pm k x)$ with $x$ one of the primes $p$ or $q$, then we can't write it as $(r-1, r+1)$. 
Since all the Fourier coefficients of the Eisenstein series are real-valued, an argument similar to one in [Merel 1996b, p. 19] shows that $F_{E_{N}}\left(s_{k} x+1,1\right)=$ $F_{E_{N}}\left(-s_{k} x-1,1\right)$. Consider the path

$$
\left\{\frac{1}{s_{k} x+2}, \frac{-1}{s_{k} x+2}\right\}=\left\{\frac{1}{s_{k} x+2}, \frac{1}{s_{k} x}\right\}+\left\{\frac{1}{s_{k} x}, \frac{-1}{s_{k} x}\right\}+\left\{\frac{-1}{s_{k} x}, \frac{-1}{s_{k} x+2}\right\} .
$$

The rational number $1 /\left(s_{k} x\right)$ corresponds to a point of $P_{-}$in the Riemann surface $X_{\Gamma}$. The differential form $k^{*} \omega_{E_{N}}$ has no zeros and poles on $P_{-}$. We deduce that

$$
\begin{aligned}
\int_{1 /\left(s_{k} x+2\right)}^{-1 /\left(s_{k} x+2\right)} & k^{*}\left(\omega_{E_{N}}\right) \\
= & \int_{1 /\left(s_{k} x+2\right)}^{1 /\left(s_{k} x\right)} k^{*}\left(\omega_{E_{N}}\right)+\int_{1 /\left(s_{k} x\right)}^{-1 /\left(s_{k} x\right)} k^{*}\left(\omega_{E_{N}}\right)+\int_{-1 /\left(s_{k} x\right)}^{-1 /\left(s_{k} x+2\right)} k^{*}\left(\omega_{E_{N}}\right) \\
= & 2 F_{N}\left(s_{k} x+1,1\right)+\int_{1 /\left(s_{k} x\right)}^{-1 /\left(s_{k} x\right)} k^{*}\left(\omega_{E_{N}}\right) .
\end{aligned}
$$

Let $\gamma_{1}^{x, k}$ and $\gamma_{2}^{x, k}$ be two matrices in $\Gamma$ such that $\gamma_{1}^{x, k}\left(1 /\left(s_{k} x+2\right)\right)=-1 /\left(s_{k} x+2\right)$ and $\gamma_{2}^{x, k}\left(1 /\left(s_{k} x\right)\right)=-1 /\left(s_{k} x\right)$. Then

$$
2 F_{N}\left(s_{k} x+1,1\right)=\int_{1 /\left(s_{k} x+2\right)}^{\gamma_{1}^{x, k}\left(1 /\left(s_{k} x+2\right)\right)} k^{*}\left(\omega_{E_{N}}\right)-\int_{1 /\left(s_{k} x\right)}^{\gamma_{2}^{x, k}\left(1 /\left(s_{k} x\right)\right)} k^{*}\left(\omega_{E_{N}}\right) .
$$

We now prove that $\int_{1 /\left(s_{k} x\right)}^{\gamma_{2}^{x, k}\left(1 /\left(s_{k} x\right)\right)} k^{*}\left(\omega_{E_{N}}\right)$ is independent of the choice of the matrices $\gamma_{2}^{x, k} \in \Gamma$ that take $1 /\left(s_{k} x\right)$ to $-1 /\left(s_{k} x\right)$. Suppose $\gamma_{2}^{x, k}$ and $\gamma_{2}^{\prime x, k}$ are two matrices such that $\gamma_{2}^{x, k}\left(1 /\left(s_{k} x\right)\right)=\gamma_{2}^{\prime x, k}\left(1 /\left(s_{k} x\right)\right)=-1 /\left(s_{k} x\right)$. Since $\gamma_{2}^{x, k} \in \Gamma$,

$$
\varphi_{E_{N}}\left(\gamma_{2}^{x, k}\right)=\int_{1 /\left(s_{k} x\right)}^{\gamma_{2}^{x, k}\left(1 /\left(s_{k} x\right)\right)} k^{*}\left(\omega_{E_{N}}\right)
$$

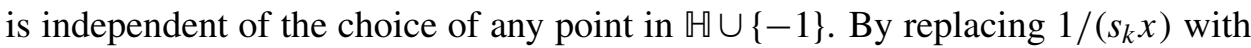
$\left(\gamma_{2}^{x, k}\right)^{-1}\left(\gamma_{2}^{\prime x, k}\right)\left(1 /\left(s_{k} x\right)\right)$, we get that the above integral is the same as

$$
\int_{1 /\left(s_{k} x\right)}^{\gamma_{2}^{\prime x, k}\left(1 /\left(s_{k} x\right)\right)} k^{*}\left(\omega_{E_{N}}\right)
$$

and the integral is independent of the choice of exceptional matrices. Similarly, we can prove that

$$
\int_{1 /\left(s_{k} x+2\right)}^{\gamma^{x, k}\left(1 /\left(s_{k} x+2\right)\right)} k^{*}\left(\omega_{E_{N}}\right)
$$

is also independent of the choice of the matrices that take $1 /\left(s_{k} x+2\right)$ to $-1 /\left(s_{k} x+2\right)$. Since we have already written down two matrices $\gamma_{1}^{x, k}$ and $\gamma_{2}^{x, k}$ in $\Gamma$ such that 
$\gamma_{1}^{x, k}\left(1 /\left(s_{k} x+2\right)\right)=-1 /\left(s_{k} x+2\right)$ and $\gamma_{2}^{x, k}\left(1 /\left(s_{k} x\right)\right)=-1 /\left(s_{k} x\right)$, we use these matrices to find those integrals.

The above calculation shows that

$2 \pi_{E_{N}}\left(\gamma_{1}^{x, k}\right)-\pi_{E_{N}}\left(h \gamma_{1}^{x, k} h^{-1}\right)=2 F_{N}\left(s_{k} x+1,1\right)+2 \pi_{E_{N}}\left(\gamma_{2}^{x, k}\right)-\pi_{E_{N}}\left(h \gamma_{2}^{x, k} h^{-1}\right)$.

We get

$$
\begin{aligned}
& F_{E_{N}}\left(s_{k} x+1,1\right) \\
& \quad=\frac{1}{2}\left(2 \pi_{E_{N}}\left(\gamma_{1}^{x, k}\right)-\pi_{E_{N}}\left(h \gamma_{1}^{x, k} h^{-1}\right)-2 \pi_{E}\left(\gamma_{2}^{x, k}\right)+\pi_{E}\left(h \gamma_{2}^{x, k} h^{-1}\right)\right) \\
& \quad=6\left(P_{N}\left(\gamma^{x, k}\right)-P_{N}\left(\gamma_{2}^{x, k}\right)\right) .
\end{aligned}
$$

Since $F_{E_{N}}\left(1+s_{k} x, 1\right)=-F_{E_{N}}\left(1,-1-s_{k} x\right)$, the above equation determines the Eisenstein elements for the Eisenstein series $E_{N}$ completely.

From the above lemma, we conclude that $6 F_{N}(g)=F_{E_{N}}(g)$.

Lemma 30. For $E_{N} \in E_{2}\left(\Gamma_{0}(p q)\right)$, consider the element $\mathcal{E}_{E_{N}}^{0} \in \mathrm{H}_{1}\left(X_{\Gamma}-P_{-}, P_{+}, \mathbb{Z}\right)$ defined by $\mathcal{E}_{E_{N}}^{0}=\sum_{g \in \mathbb{P}^{1}(\mathbb{Z} / p q \mathbb{Z})} F_{E_{N}}(g) \xi^{0}(g)$. For all $c \in \mathrm{H}_{1}\left(X_{\Gamma}-P_{+}, P_{-}, \mathbb{Z}\right)$, we have $\mathcal{E}_{E_{N}}^{0} \circ c=\varphi_{E_{N}}(c)$.

Proof. By Theorem 19, we can write the even Eisenstein element uniquely as

$$
\sum_{g \in \mathbb{P}^{1}(\mathbb{Z} / p q \mathbb{Z})} H_{E_{N}}(g) \xi^{0}(g) .
$$

By the same theorem, $[g]_{0} \circ[h]^{0}=1$ if and only if $\Gamma g=\Gamma h$. The functions $H_{E_{N}}$ and $F_{E_{N}}$ coincide since

$$
H_{E_{N}}(g)=\sum_{g \in \mathbb{P}^{1}(\mathbb{Z} / p q \mathbb{Z})} H_{E_{N}}(g) \xi^{0}(g) \circ \xi_{0}(g)=\mathcal{E}_{E_{N}}^{0} \circ \xi_{0}(g)=F_{E_{N}}(g) .
$$

For the modular curve $X_{\Gamma}$, we have a similar short exact sequence

$$
0 \rightarrow \mathrm{H}_{1}\left(X_{\Gamma}-P_{-}, \mathbb{Z}\right) \rightarrow \mathrm{H}_{1}\left(X_{\Gamma}-P_{-}, P_{+}, \mathbb{Z}\right) \stackrel{\delta^{0}}{\rightarrow} \mathbb{Z}^{P_{+}} \rightarrow \mathbb{Z} \rightarrow 0 .
$$

The boundary map $\delta^{0}$ takes a geodesic, joining the points $r$ and $s$ of $P_{+}$to the formal symbol $[r]-[s]$.

\section{Eisenstein elements and winding elements for $\Gamma_{0}(p q)$}

7.1. Eisenstein elements for $\boldsymbol{\Gamma}_{\mathbf{0}}(\boldsymbol{p q})$. We first prove an elementary number theoretic lemma. Recall, $l$ and $m$ are two unique integers such that $l q+m p \equiv 1(\bmod p q)$ with $1 \leq l \leq p-1$ and $1 \leq m \leq q-1$.

Lemma 31. For all $k$ with $1 \leq k \leq q-1$, we can choose an integer $s(k) \in \mathbb{Z} / q \mathbb{Z}$ such that

$$
(k p,-1)=(p, s(k) p-1)
$$


in $\mathbb{P}^{1}(\mathbb{Z} / p q \mathbb{Z})$. The map $k \rightarrow s(k)$ is a bijection $(\mathbb{Z} / q \mathbb{Z})^{*} \rightarrow \mathbb{Z} / q \mathbb{Z}-\{\bar{m}\}$.

Proof. For all $k$ with $1 \leq k \leq q-1$, let $k^{\prime}$ be the inverse of $k$ in $(\mathbb{Z} / q \mathbb{Z})^{*}$. By the Chinese remainder theorem, we choose a unique $x$ with $1 \leq x \leq p q-1$ such that $x \equiv-1(\bmod p)$ and $x \equiv-k^{\prime}(\bmod q)$. Observe that $x$ is coprime to both $p$ and $q$. We write $x=s(k) p-1$ for a unique $s(k)$ with $0 \leq s(k) \leq q-1$. Since $\Gamma_{0}(p q) \backslash \mathrm{SL}_{2}(\mathbb{Z}) \cong \mathbb{P}^{1}(\mathbb{Z} / p q \mathbb{Z})$, we deduce that $(k p,-1)=(x k p,-x)=$ $(-p,-x)=(p, x)=(p, s(k) p-1)$ in $\mathbb{P}^{1}(\mathbb{Z} / p q \mathbb{Z})$.

Consider the map $(\mathbb{Z} / q \mathbb{Z})^{*} \rightarrow \mathbb{Z} / q \mathbb{Z}$ given by $k \rightarrow s(k)$. If $l q+m p \equiv 1(\bmod p q)$ then $m$ is not in the image. This map is one-to-one since $s(k)=s(h)$ implies $k \equiv h(\bmod q)$. Thus the map $(\mathbb{Z} / q \mathbb{Z})^{*} \rightarrow \mathbb{Z} / q \mathbb{Z}-\{\bar{m}\} k \rightarrow s(k)$ is a bijection.

For all $t$ coprime to $p q$, consider the set $V$ of all matrices of the form $\alpha_{t}$.

Proposition 32. The boundary of any element

$$
X=\sum_{g \in \mathbb{P}^{1}(\mathbb{Z} / p q \mathbb{Z})} F(g)[g]^{*}
$$

in $\mathrm{H}_{1}\left(X_{0}(p q)-R \cup I, \partial\left(X_{0}(p q)\right), \mathbb{Z}\right)$ is of the form

$$
\delta(X)=A(X)\left[\frac{1}{p}\right]+B(X)\left[\frac{1}{q}\right]+C(X)[\infty]-(A(X)+B(X)+C(X))[0]
$$

with

$$
\begin{aligned}
& A(X)=\sum_{k=0}^{q-1}\left(F\left(\beta_{k}\right)-F\left(\beta_{k} S\right)\right), \\
& B(X)=\sum_{i=0}^{p-1}\left(F\left(\gamma_{i}\right)-F\left(\gamma_{i} S\right)\right), \\
& C(X)=F(0,1)-F(1,0) .
\end{aligned}
$$

Proof. Choose an explicit coset representative of $\Gamma_{0}(p q) \backslash \mathrm{SL}_{2}(\mathbb{Z})$ (see Lemma 8) and write

$$
\begin{aligned}
X=C(X)[I]^{*} & +\sum_{\alpha_{t} \in V} F(1, t)\left[\alpha_{t}\right]^{*}+\sum_{k=1}^{q-1} F(1, k p)\left[\alpha_{k p}\right]^{*} \\
& +\sum_{k=1}^{p-1} F(1, k q)\left[\alpha_{k q}\right]^{*}+\sum_{i=0}^{q-1} F(p, i p-1)\left[\beta_{i}\right]^{*}+\sum_{j=0}^{p-1} F(q, j q-1)\left[\beta_{j}\right]^{*} .
\end{aligned}
$$


According to Lemma 31 for $1 \leq k \leq q-1$, we have $\alpha_{k p} S=Z \beta_{s(k)}$ for some $Z \in \Gamma_{0}(p q)$. We deduce that

$$
\begin{aligned}
\sum_{k=1}^{q-1} F(1, k p)\left[\alpha_{k p}\right]^{*}+ & \sum_{i=0}^{q-1} F(p, i p-1)\left[\beta_{i}\right]^{*} \\
& =\sum_{k=1}^{q-1}\left(F(1, k p)\left[\alpha_{k p}\right]^{*}+F(k p,-1)\left[\alpha_{k p} S\right]^{*}\right)+F\left(\beta_{m}\right)\left[\beta_{m}\right]^{*}
\end{aligned}
$$

and

$$
\begin{aligned}
\sum_{k=1}^{p-1} F(1, k q)\left[\alpha_{k q}\right]^{*}+ & \sum_{j=0}^{p-1} F(q, j q-1)\left[\gamma_{j}\right]^{*} \\
& =\sum_{k=1}^{p-1}\left(F(1, k q)\left[\alpha_{k q}\right]^{*}+F(k q,-1)\left[\alpha_{k q} S\right]^{*}\right)+F\left(\gamma_{l}\right)\left[\gamma_{l}\right]^{*} .
\end{aligned}
$$

A small check shows that $\delta\left(\left[\alpha_{k p}\right]^{*}\right)=\delta\left(\left[\alpha_{p}\right]^{*}\right)$ and $\delta\left(\left[\alpha_{k p}\right]^{*}\right)=-\delta\left(\left[\alpha_{k p} S\right]^{*}\right)$.

We now calculate $\delta\left(\left[\beta_{m}\right]^{*}\right)$ and $\delta\left(\left[\gamma_{l}\right]^{*}\right)$. Since $l q+m p \equiv 1(\bmod p q)$ and $-I \in \Gamma_{0}(p q)$, we get

$$
\left(\begin{array}{cc}
1-q(l-1) & m(l-1) \\
(l-1) p q & 1+l q(l-1)
\end{array}\right)\left(\begin{array}{cc}
m & -l \\
q & p
\end{array}\right)=\gamma \beta_{m} S
$$

and

$$
\left(\begin{array}{cc}
1-p(m+1) & -l(m+1) \\
(1+m) p q & 1-m p(l+m)
\end{array}\right)\left(\begin{array}{cc}
m & -l \\
q & p
\end{array}\right)=\left(\begin{array}{cc}
-1 & -l \\
q & -m p
\end{array}\right)=\gamma_{l}
$$

for some $\gamma \in \Gamma_{0}(p q)$, and hence we have $\Gamma_{0}(p q) \beta_{m} S=\gamma_{l}$. From $\delta\left(\left[\beta_{m}\right]^{*}\right)=$ $\delta\left(\left[\alpha_{q}\right]^{*}-\left[\alpha_{p}\right]^{*}\right)$ and $\delta\left(\left[\gamma_{l}\right]^{*}\right)=\delta\left(\left[\alpha_{p}\right]^{*}-\left[\alpha_{q}\right]^{*}\right)$, it is easy to see that

$$
\begin{aligned}
\delta\left(\sum_{k=1}^{q-1} F(1, k p)\left[\alpha_{k p}\right]^{*}\right. & \left.+\sum_{i=0}^{q-1} F(p, j p-1)\left[\beta_{j}\right]^{*}\right) \\
& =\sum_{k=1}^{q-1}(F(1, k p)-F(k p,-1)) \delta\left(\left[\alpha_{p}\right]^{*}\right)+F\left(\beta_{m}\right) \delta\left(\left[\beta_{m}\right]^{*}\right)
\end{aligned}
$$

and

$$
\begin{aligned}
\delta\left(\sum_{k=1}^{p-1} F(1, k q)\left[\alpha_{k q}\right]^{*}+\sum_{j=0}^{p-1} F(q, j q-1)\left[\gamma_{j}\right]^{*}\right) & \\
= & \sum_{k=1}^{p-1}(F(1, k q)-F(k q,-1)) \delta\left(\left[\alpha_{q}\right]^{*}\right)+F(q, l q-1) \delta\left(\left[\gamma_{l}\right]^{*}\right) .
\end{aligned}
$$


We have

$$
\begin{aligned}
F(p, m p-1) \delta\left(\left[\beta_{m}\right]^{*}\right)+F(q, l q-1) \delta\left(\left[\gamma_{l}\right]^{*}\right) & \\
= & \left(F\left(\beta_{m}\right)-F\left(\beta_{m} S\right)\right)\left(\delta\left(\left[\alpha_{q}\right]^{*}\right)-\delta\left(\left[\alpha_{p}\right]^{*}\right)\right) .
\end{aligned}
$$

Recall, $\delta\left(\left[\alpha_{p}\right]^{*}\right)=[0]-[1 / p]$ and $\delta\left(\left[\alpha_{q}\right]^{*}\right)=[0]-[1 / q]$. The above calculation shows that

$$
\delta(X)=C(X) \delta\left([I]^{*}\right)+A(X) \delta\left(\left[\alpha_{p}\right]^{*}\right)+B(X) \delta\left(\left[\alpha_{q}\right]^{*}\right)
$$

with

$$
\begin{aligned}
& A(X)=\sum_{k=0}^{q-1}(F(p, k p-1)-F(k p-1,-p)), \\
& B(X)=\sum_{m=0}^{p-1}\left(F\left(\gamma_{l}^{\prime}\right)-F\left(\gamma_{l}^{\prime} S\right)\right), \\
& C(X)=F(I)-F(S) .
\end{aligned}
$$

We also prove a similar proposition for $\Gamma \subset \Gamma(2)$.

Proposition 33. The boundary of any element

$$
X=\sum_{g \in \mathbb{P}^{1}(\mathbb{Z} / p q \mathbb{Z})} F(g) \xi^{0}(g)
$$

in $\mathrm{H}_{1}\left(X_{\Gamma}-P_{-}, P_{+}, \mathbb{Z}\right)$ is of the form

$$
\delta^{0}(X)=A^{\prime}(X)\left[\frac{1}{p}\right]+B^{\prime}(X)\left[\frac{1}{q}\right]+C^{\prime}(X)[\infty]-\left(A^{\prime}(X)+B^{\prime}(X)+C^{\prime}(X)\right)[0]
$$

with

$$
\begin{aligned}
& A^{\prime}(X)=\sum_{k=0}^{q-1} F\left(\beta_{k}^{\prime}\right)-\left(\sum_{k=1}^{q-1} F\left(\alpha_{k p}^{\prime}\right)\right)-F\left(\gamma_{l}^{\prime}\right), \\
& B^{\prime}(X)=\sum_{i=0}^{p-1} F\left(\gamma_{i}^{\prime}\right)-\left(\sum_{k=1}^{p-1} F\left(\alpha_{k q}^{\prime}\right)\right)-F\left(\beta_{m}^{\prime}\right), \\
& C^{\prime}(X)=F(0,1)-F\left(\alpha_{p q}^{\prime}\right) .
\end{aligned}
$$

Proof. This is a straightforward calculation using the coset representatives of $\Gamma \backslash \Gamma(2)$ (see Lemma 16).

Proposition 34. For $E \in \mathbb{E}_{p q}$, the boundaries of almost Eisenstein elements $\mathcal{E}_{E}^{\prime}$ in $\mathrm{H}_{1}\left(X_{0}(p q)-R \cup I, \partial\left(X_{0}(p q)\right), \mathbb{Z}\right)$ corresponding to the Eisenstein series $E$ are $-\delta(E)$ (Section 4$)$. 
Proof. For $E \in \mathbb{E}_{p q}$, let $\mathcal{E}_{E}^{\prime}=\sum_{g \in \mathbb{P}^{1}(\mathbb{Z} / p q \mathbb{Z})} G_{E}(g)[g]^{*}$ be the almost Eisenstein element. According to Proposition 32, we need to calculate $A\left(\mathcal{E}_{E}^{\prime}\right), B\left(\mathcal{E}_{E}^{\prime}\right)$ and $C\left(\mathcal{E}_{E}^{\prime}\right)$.

For all $0 \leq k<q-1, \beta_{k} T=\beta_{k+1}$ and $\beta_{q-1} T=\gamma \beta_{0}$ with

$$
\gamma=\left(\begin{array}{cc}
1+p q & q \\
-q p^{2} & 1-q p
\end{array}\right)
$$

We have an inclusion $\mathrm{H}_{1}\left(Y_{0}(p q), \mathbb{Z}\right) \rightarrow \mathrm{H}_{1}\left(Y_{0}(p q), R \cup I, \mathbb{Z}\right)$. Since $\left\{\rho^{*}, \gamma \rho^{*}\right\}=$ $\left\{\beta_{0} \rho^{*}, \gamma \beta_{0} \rho^{*}\right\}=-\sum_{k=0}^{q-1}\left\{\beta_{k} \rho, \beta_{k} \rho^{*}\right\}$, we deduce that

$$
\begin{aligned}
\pi_{E}(\gamma) & =\int_{z_{0}}^{\gamma z_{0}} E(z) d z \\
& =\mathcal{E}_{E}^{\prime} \circ\left\{z_{0}, \gamma z_{0}\right\} \\
& =-\mathcal{E}_{E}^{\prime} \circ\left(\sum_{k=0}^{q-1}\left\{\beta_{k} \rho, \beta_{k} \rho^{*}\right\}\right) \\
& =-\sum_{k=0}^{q-1} \mathcal{E}_{E}^{\prime} \circ\left\{\beta_{k} \rho, \beta_{k} \rho^{*}\right\} .
\end{aligned}
$$

Applying Corollary 6, we have

$$
\sum_{k=0}^{q-1} \mathcal{E}_{E}^{\prime} \circ\left\{\beta_{k} \rho, \beta_{k} \rho^{*}\right\}=\sum_{k=0}^{q-1}\left(G_{E}\left(\beta_{k}\right)-G_{E}\left(\beta_{k} S\right)\right)=-A\left(\mathcal{E}_{E}^{\prime}\right) .
$$

Hence, we prove that $A\left(\mathcal{E}_{E}^{\prime}\right)=-\pi_{E}(\gamma)$. By interchanging $p$ and $q$, we have $B\left(\mathcal{E}_{E}^{\prime}\right)=-\pi_{E}\left(\gamma_{0}\right)$ for

$$
\gamma_{0}=\left(\begin{array}{cc}
1+p q & p \\
-p q^{2} & 1-q p
\end{array}\right)
$$

We now calculate $\pi_{E}(\gamma)$ and $\pi_{E}\left(\gamma_{0}\right)$ using [Stevens 1985]. Recall, $1 / p$ is a cusp with $e_{\Gamma_{0}(p q)}(1 / p)=q$. Consider the matrices

$$
x=\left(\begin{array}{cc}
1 & -q \\
-p & 1+q p
\end{array}\right) \quad \text { and } \quad y=\left(\begin{array}{cc}
1 & -p \\
-q & 1+q p
\end{array}\right) .
$$

One can easily check that $x\left(\begin{array}{ll}1 & q \\ 0 & 1\end{array}\right) x^{-1}=\gamma$ and $y\left(\begin{array}{ll}1 & p \\ 0 & 1\end{array}\right) y^{-1}=\gamma_{0}$. Notice that $x(i \infty)=$ $\Gamma_{0}(p q) 1 / p$ and $y(i \infty)=\Gamma_{0}(p q) 1 / q$. By [Stevens 1985, p. 524], we deduce that $\pi_{E}(\gamma)=e_{\Gamma_{0}(p q)}(1 / q) a_{0}(E[1 / p])$ and $\pi_{E_{p q}}\left(\gamma_{0}\right)=e_{\Gamma_{0}(p q)}(1 / p) a_{0}(E[1 / p])$.

According to Proposition 32, the boundary of the almost Eisenstein element corresponding to an Eisenstein series $E$ is

$$
\delta\left(\mathcal{E}_{E}^{\prime}\right)=A\left(\mathcal{E}_{E}^{\prime}\right)\left[\frac{1}{p}\right]+B\left(\mathcal{E}_{E}^{\prime}\right)\left[\frac{1}{q}\right]+C\left(\mathcal{E}_{E}^{\prime}\right)[\infty]-\left(A\left(\mathcal{E}_{E}^{\prime}\right)+B\left(\mathcal{E}_{E}^{\prime}\right)+C\left(\mathcal{E}_{E}^{\prime}\right)\right)[0]
$$


with $A\left(\mathcal{E}_{E}^{\prime}\right)=q a_{0}(E[1 / p]), B\left(\mathcal{E}_{E}^{\prime}\right)=p a_{0}(E[1 / q])$ and $C\left(\mathcal{E}_{E}^{\prime}\right)=-(F(I)-F(S))$. Applying Corollary 6 again, we deduce that $F(I)-F(S)=\int_{\rho}^{\rho^{*}} E(z) d z=-a_{0}(E)$. For $E \in E_{2}\left(\Gamma_{0}(p q)\right)$, the boundary of $E$ is

$$
\begin{aligned}
\delta(E)= & a_{0}(E)([\infty]-[0]) \\
& +q a_{0}\left(E\left[\frac{1}{p}\right]\right)\left(\left[\frac{1}{p}\right]-[0]\right)+p a_{0}\left(E\left[\frac{1}{q}\right]\right)\left(\left[\frac{1}{q}\right]-[0]\right) \\
=\delta\left(\mathcal{E}_{E}^{\prime}\right) . & \square
\end{aligned}
$$

Let $\beta$ and $h$ be the matrices $\left(\begin{array}{ll}1 & 2 \\ 0 & 1\end{array}\right)$ and $\left(\begin{array}{ll}1 & 1 \\ 0 & 2\end{array}\right)$ respectively. Let

$$
\pi_{*}: \mathrm{H}_{1}\left(X_{\Gamma}-P_{-}, P_{+}, \mathbb{Z}\right) \rightarrow \mathrm{H}_{1}\left(X_{0}(p q)-R \cup I, \partial\left(X_{0}(p q)\right), \mathbb{Z}\right)
$$

be the isomorphism defined by $\pi_{*}\left(\xi_{0}(g)\right)=[g]^{*}$ [Merel 1995, Corollary 1]. It is easy to see that $\delta\left(\pi_{*}(X)\right)=\delta^{0}(X)$ for all $X \in \mathrm{H}_{1}\left(X_{\Gamma}-P_{-}, P_{+}, \mathbb{Z}\right)$.

Proposition 35. For all $E \in \mathbb{E}_{p q}$, let $\mathcal{E}_{E}^{0}$ denote the even Eisenstein element in $\mathrm{H}_{1}\left(X_{\Gamma}-P_{-}, P_{+}, \mathbb{Z}\right)$ (Section 6). The boundary of the modular symbol $\pi_{*}\left(\mathcal{E}_{E}^{0}\right)$ is $-6 \delta(E)$.

Proof. By Theorem 18, we can explicitly write down the even Eisenstein element $\mathcal{E}_{E}^{0}$ in the relative homology group $\mathrm{H}_{1}\left(X_{\Gamma}-P_{-}, P_{+}, \mathbb{Z}\right)$ as

$$
\mathcal{E}_{E}^{0}=\sum_{g \in \mathbb{P}^{1}(\mathbb{Z} / p q \mathbb{Z})} F_{E}(g) \xi_{0}(g) .
$$

According to Proposition 33 , we need to calculate $A^{\prime}\left(\mathcal{E}_{E}^{0}\right), B^{\prime}\left(\mathcal{E}_{E}^{0}\right)$ and $C^{\prime}\left(\mathcal{E}_{E}^{0}\right)$. For $0 \leq k<q-2$, we have $\beta_{k}^{\prime} \beta=\beta_{k+2}^{\prime}$. A small check shows that $\beta_{q-1}^{\prime} \beta=\beta_{1}^{\prime}$ and $\beta_{q-2}^{\prime} \beta=\gamma^{\prime} \beta_{0}^{\prime}$ with

$$
\gamma^{\prime}=\left(\begin{array}{cc}
1+2 p q(1+q) & 2 q \\
-2 q(p+p q)^{2} & 1-2 p q(1+q)
\end{array}\right) \in \Gamma
$$

As a homology class in $\mathrm{H}_{1}\left(X_{\Gamma}-P_{+}, P_{-}, \mathbb{Z}\right)$, we have

$$
\begin{aligned}
\left\{-1, \gamma^{\prime}(-1)\right\} & =\left\{\beta_{0}^{\prime}(-1), \gamma^{\prime} \beta_{0}^{\prime}(-1)\right\} \\
& =-\sum_{k=0}^{q-1}\left\{\beta_{k}^{\prime}(1), \beta_{k}^{\prime}(-1)\right\} \\
& =\sum_{k=0}^{q-1}\left\{\beta_{k}^{\prime}(-1), \beta_{k}^{\prime}(1)\right\} .
\end{aligned}
$$


By the definition of the even Eisenstein elements, we conclude that

$$
\begin{aligned}
\int_{z_{0}}^{\gamma^{\prime} z_{0}} k^{*}\left(\omega_{E}\right) & =\mathcal{E}_{E}^{0} \circ\left\{z_{0}, \gamma^{\prime} z_{0}\right\} \\
& =-\mathcal{E}_{E}^{0} \circ \sum_{k=0}^{q-1}\left(\beta_{k}^{\prime}(1), \beta_{k}^{\prime}(-1)\right) \\
& =-\sum_{k=0}^{q-1} \mathcal{E}_{E}^{0} \circ\left\{\beta_{k}^{\prime}(1), \beta_{k}^{\prime}(-1)\right\} .
\end{aligned}
$$

It is easy to see that $h A S B h^{-1} \in \mathrm{SL}_{2}(\mathbb{Z})$ for all $A, B \in \Gamma(2)$. Since $\left[\alpha_{k q}^{\prime} S\right]=\left[\gamma_{s(k)}^{\prime}\right]$ in $\mathbb{P}^{1}(\mathbb{Z} / p q \mathbb{Z})$, we have $\kappa^{\prime}=\alpha_{k q}^{\prime} S\left(\gamma_{s(k)}^{\prime}\right)^{-1} \in \Gamma_{0}(p q)$ and $h \kappa^{\prime} h^{-1} \in \Gamma_{0}(p q)$. We deduce that the differential form

$$
k^{*}\left(\omega_{E}\right)=f(z) d z=\left(2 E(z)-\frac{1}{2} E\left(\frac{1}{2}(z+1)\right)\right) d z
$$

is invariant under $\kappa^{\prime}$. According to the above argument,

$$
\begin{aligned}
F_{E}\left(\alpha_{k q}^{\prime}\right) & =\int_{\alpha_{k q}^{\prime}(1)}^{\alpha_{k q}^{\prime}(-1)} f(z) d z \\
& =\int_{\alpha_{k q}^{\prime} S(-1)}^{\alpha_{k q}^{\prime} S(1)} f(z) d z \\
& =-\int_{\alpha_{k q}^{\prime} S(1)}^{\alpha_{k q}^{\prime} S(-1)} f(z) d z \\
& =-\int_{\kappa^{\prime-1} \alpha_{k q}^{\prime} S(1)}^{\kappa^{\prime-1} \alpha_{k q}^{\prime} S(-1)} f\left(\kappa^{\prime} z\right) d \kappa^{\prime} z \\
& =-\int_{\gamma_{s(k)}^{\prime}(1)}^{\gamma_{s(k)}^{\prime}(-1)} f(z) d z \\
& =-F_{E}\left(\gamma_{s(k)}^{\prime}\right) .
\end{aligned}
$$

A similar calculation shows that $F_{E}\left(\gamma_{l}^{\prime}\right)=-F_{E}\left(\beta_{m}^{\prime}\right)$ and $F_{E}\left(\alpha_{k p}\right)=-F_{E}\left(\beta_{s(k)}\right)$ for some $s(k) \in(\mathbb{Z} / q \mathbb{Z})^{*}$. Applying Theorem 18, we have

$$
\sum_{k=0}^{q-1} F_{E}\left(\beta_{k}^{\prime}\right)=\sum_{k=0}^{q-1} \mathcal{E}_{E}^{0} \circ\left\{\beta_{k}^{\prime}(1), \beta_{k}^{\prime}(-1)\right\}=-\int_{z_{0}}^{\gamma^{\prime} z_{0}} k^{*}\left(\omega_{E}\right) .
$$

According to the definition of the period $\pi_{E}$ of the Eisenstein series $E(z)$ (see Section 4), we get

$$
\int_{z_{0}}^{\gamma^{\prime} z_{0}} k^{*}\left(\omega_{E}\right)=\int_{z_{0}}^{\gamma^{\prime} z_{0}}\left(2 E(z)-\frac{1}{2} E\left(\frac{1}{2}(z+1)\right)\right) d z=2 \pi_{E}\left(\gamma^{\prime}\right)-\pi_{E}\left(h \gamma^{\prime} h^{-1}\right) .
$$


We calculate $\pi_{E}\left(\gamma^{\prime}\right)$ and $\pi_{E}\left(h \gamma^{\prime} h^{-1}\right)$. From Remark 27, we see that

$$
h \gamma^{\prime} h^{-1}=\left(\begin{array}{cc}
1+z & q v^{2} \\
-4 p^{2} q(1+q)^{2} & 1-z
\end{array}\right)
$$

with $v=1-p(1+q)$ and $z=2 p q v(1+q)$. Furthermore, the matrix $h \gamma^{\prime} h^{-1}$ decomposes as

$$
h \gamma^{\prime} h^{-1}=\left(\begin{array}{cc}
1-p(1+q) & \frac{1}{2} p(1+q) \\
-2 p(1+q) & 1+p(1+q)
\end{array}\right)\left(\begin{array}{cc}
1 & q \\
0 & 1
\end{array}\right)\left(\begin{array}{cc}
1-p(1+q) & \frac{1}{2} p(1+q) \\
-2 p(1+q) & 1+p(1+q)
\end{array}\right)^{-1} .
$$

Since the matrix

$$
\left(\begin{array}{cc}
1-p(1+q) & \frac{1}{2} p(1+q) \\
-2 p(1+q) & 1+p(1+q)
\end{array}\right)^{-1}
$$

takes the cusp $i \infty$ to $1 / p$, we have $\pi_{E}\left(h \gamma^{\prime} h^{-1}\right)=q a_{0}(E[1 / p])$. We further decompose $\gamma^{\prime}$ as

$$
\left(\begin{array}{cc}
1 & -2 q \\
-p(1+q) & 1+2 p q(1+q)
\end{array}\right)\left(\begin{array}{cc}
1 & 2 q \\
0 & 1
\end{array}\right)\left(\begin{array}{cc}
1 & -2 q \\
-p(1+q) & 1+2 p q(1+q)
\end{array}\right)^{-1} .
$$

The matrix

$$
\left(\begin{array}{cc}
1 & -2 q \\
p(1+q) & 1+2 p q(1+q)
\end{array}\right)
$$

takes the cusp $i \infty$ to $1 / p$. We see that $\pi_{E}\left(\gamma^{\prime}\right)=2 q a_{0}(E[1 / p])$ and $\int_{z_{0}}^{\gamma^{\prime} z_{0}} k^{*}\left(\omega_{E}\right)=$ $3 a_{0}(E[1 / p])$. A simple calculation shows that

$$
A^{\prime}\left(\mathcal{E}_{E}^{0}\right)=\sum_{k=0}^{q-1} F_{E}\left(\beta_{k}^{\prime}\right)-\sum_{k=0}^{q-1} F_{E}\left(\alpha_{k p}^{\prime}\right)-F_{E}\left(\gamma_{m}^{\prime}\right)=2 \sum_{k=0}^{q-1} F_{E}\left(\beta_{k}^{\prime}\right)=-6 a_{0}\left(E\left[\frac{1}{p}\right]\right) .
$$

By interchanging $p$ and $q$, we get $B^{\prime}\left(\mathcal{E}_{E}^{0}\right)=-6 a_{0}(E[1 / q])$. Since $\alpha_{p q}^{\prime} S \in \Gamma_{0}(p q)$, a calculation similar to (7-2) shows that

$$
\begin{aligned}
F_{E}(I) & =-F_{E}\left(\alpha_{p q}\right) \\
& =\int_{1}^{-1}\left(2 E(z)-\frac{1}{2} E\left(\frac{1}{2}(z+1)\right)\right) d z \\
& =-\int_{-1}^{\beta(-1)}\left(2 E(z)-\frac{1}{2} E\left(\frac{1}{2}(z+1)\right)\right) d z \\
& =-3 a_{0}(E) .
\end{aligned}
$$

We conclude that $C^{\prime}\left(\mathcal{E}_{E}^{0}\right)=F_{E}(I)-F_{E}\left(\alpha_{p q}\right)=-6 a_{0}(E)$ and hence $\delta^{0}\left(\mathcal{E}_{E}^{0}\right)=$ $\delta\left(\mathcal{E}_{E}^{0}\right)=-6 \delta(E)$. 
The inclusion map

$$
i:\left(X_{0}(p q)-R \cup I, \partial\left(X_{0}(p q)\right)\right) \rightarrow\left(X_{0}(p q), \partial\left(X_{0}(p q)\right)\right)
$$

induces an onto map

$$
i_{*}: \mathrm{H}_{1}\left(X_{0}(p q)-R \cup I, \partial\left(X_{0}(p q)\right), \mathbb{Z}\right) \rightarrow \mathrm{H}_{1}\left(X_{0}(p q), \partial\left(X_{0}(p q)\right), \mathbb{Z}\right)
$$

with $i_{*}\left([g]^{*}\right)=\xi(g)$. Note that $\delta\left([g]^{*}\right)=[g 0]-[g \infty]=\delta^{\prime}(\xi(g))=\delta^{\prime}\left(i_{*}\left([g]^{*}\right)\right)$. From Section 3.4, we have that $\delta(c)=\delta^{\prime}\left(i_{*}(c)\right)$ for all homology classes $c \in$ $\mathrm{H}_{1}\left(X_{0}(p q)-R \cup I, \partial\left(X_{0}(p q)\right), \mathbb{Z}\right)$.

Lemma 36. The integrals of every holomorphic differential on $X_{0}(p q)$ over $i_{*}\left(\mathcal{E}_{E}^{\prime}\right)$ and $i_{*} \pi_{*}\left(\mathcal{E}_{E}^{0}\right)$ are zero.

Proof. The proof is a straightforward generalization of [Merel 1996b, Lemma 5].

We now prove the main theorem.

Proof of Theorem 1. By [Merel 1995, Corollary 3], we obtain $i_{*}\left(\mathcal{E}_{E}^{\prime}\right) \circ c=\mathcal{E}_{E}^{\prime} \circ i^{*} c=$ $\int_{c} i_{*}(E(z) d z)$. Hence, $i_{*}\left(\mathcal{E}_{E}^{\prime}\right)$ is the Eisenstein element inside the space of modular symbols corresponding to $E$. By Propositions 34 and 35, the boundary of $\pi_{*}\left(\mathcal{E}_{E}^{0}\right)$ is the same as the boundary of $6 i_{*}\left(\mathcal{E}_{E}^{\prime}\right)$.

There is a nondegenerate bilinear pairing $S_{2}\left(\Gamma_{0}(p q)\right) \times \mathrm{H}_{1}\left(X_{0}(p q), \mathbb{R}\right) \rightarrow \mathbb{C}$ given by $(f, c)=\int_{c} f(z) d z$. Hence, the integrals of holomorphic differentials over $\mathrm{H}_{1}\left(X_{0}(p q), \mathbb{Z}\right)$ are not always zero. By Lemma 36 , the integrals of holomorphic differentials over $i_{*}\left(\mathcal{E}_{E}^{\prime}\right)$ and $i_{*}\left(\pi_{*}\left(\mathcal{E}_{E}^{0}\right)\right)$ are always zero. We deduce that

$$
\mathcal{E}_{E}=i_{*}\left(\mathcal{E}_{E}^{\prime}\right)=\frac{1}{6} i_{*} \pi_{*}\left(\mathcal{E}_{E}^{0}\right)=\frac{1}{6} \sum_{g \in \mathbb{P}^{(\mathbb{Z} / p q \mathbb{Z})}} F_{E}(g) \xi(g)
$$

for $E \in \mathbb{E}_{p q}$. Since $F_{N}(g)=\frac{1}{6} F_{E_{N}}(g)$, we obtain the theorem.

7.2. Winding elements of level pq. Recall the concept of the winding element.

Definition 37 (winding element). Let $\{0, \infty\}$ denote the projection of the path from 0 to $\infty$ in $\mathbb{U} \cup \mathbb{P}^{1}(\mathbb{Q})$ to $X_{0}(p q)(\mathbb{C})$. We have an isomorphism $\mathrm{H}_{1}\left(X_{0}(p q), \mathbb{Z}\right) \otimes \mathbb{R}=$ $\operatorname{Hom}_{\mathbb{C}}\left(\mathrm{H}^{0}\left(X_{0}(p q), \Omega^{1}\right), \mathbb{C}\right)$. Let $e_{p q} \in \mathrm{H}_{1}\left(X_{0}(p q), \mathbb{R}\right)$ correspond to the homomorphism $\omega \rightarrow-\int_{0}^{\infty} \omega$. The modular symbol $e_{p q}$ is called the winding element.

The winding elements are the elements of the space of modular symbols whose annihilators define ideals of the Hecke algebras with the $L$-functions of the corresponding quotients of the Jacobian nonzero. In this paper, we find an explicit expression of the winding element. Let $e_{p q} \in \mathrm{H}_{1}\left(X_{0}(p q), \mathbb{Z}\right) \otimes \mathbb{R}$ be the winding element. The following lemma will help us write down the winding element 
explicitly. Since $\sum_{x \in \partial\left(X_{0}(p q)\right)} e_{\Gamma_{0}(p q)}(x) a_{0}(E[x])=0$, we write $\delta(E)=a_{0}(E)(\{\infty\}-\{0\})+q a_{0}\left(E\left[\frac{1}{p}\right]\right)\left(\left\{\frac{1}{p}\right\}-\{0\}\right)+p a_{0}\left(E\left[\frac{1}{q}\right]\right)\left(\left\{\frac{1}{q}\right\}-\{0\}\right)$.

Lemma 38. The constant Fourier coefficients of $E_{p q}$ at cusps $0,1 / p, 1 / q$ and $\infty$ are $\frac{1}{24}(1-p q) /(p q), 0,0$ and $\frac{1}{24}(p q-1)$ respectively.

Proof. We first prove that the constant coefficient for the Fourier expansion of $E_{p q}$ at the cusp $1 / p$ is 0 . As usual, the constant term of the Fourier expansion of $E_{p q}$ at the cusp $1 / p$ is the constant term at $\infty$ of $E_{p q}\left[\beta_{0}\right]$. Similarly, the constant term of the Fourier expansion of $E_{p q}$ at the cusp $1 / q$ is the constant term at $\infty$ of $E_{p q}\left[\gamma_{0}\right]$. Let $\Delta$ be the Ramanujan cusp form of weight 12 . We write

$$
\frac{d}{d z} \log \Delta(\beta(z))=12 \frac{d}{d z} \log (p z+1)+\frac{d}{d z} \log \Delta(z) \quad \text { for } \beta=\left(\begin{array}{ll}
1 & 0 \\
p & 1
\end{array}\right) .
$$

A simple calculation shows that

$$
\begin{aligned}
\Delta\left(\frac{p q z}{p z+1}\right) & =\Delta\left(\left(\begin{array}{ll}
q & 0 \\
1 & 1
\end{array}\right) p z\right) \\
& =\Delta\left(\left(\begin{array}{cc}
q & -1 \\
1 & 0
\end{array}\right)\left(\begin{array}{ll}
1 & 1 \\
0 & q
\end{array}\right) p z\right) \\
& =\Delta\left(\left(\begin{array}{cc}
q & -1 \\
1 & 0
\end{array}\right)\left(\frac{p z+1}{q}\right)\right) \\
& =\left(\frac{p z+1}{q}\right)^{12} \Delta\left(\frac{p z+1}{q}\right) .
\end{aligned}
$$

By taking logarithmic derivative, we deduce that

$$
\frac{d}{d z} \log \Delta\left(\begin{array}{cc}
q & -1 \\
1 & 0
\end{array}\right)\left(\frac{p z+1}{q}\right)=12 \frac{d}{d z} \log (p z+1)+\frac{d}{d z} \log \Delta\left(\frac{p z+1}{q}\right) .
$$

Since

$$
E_{p q}(z)=\frac{1}{2 \pi i} \frac{d}{d z} \log \frac{\Delta(p q z)}{\Delta(z)},
$$

the above calculation shows that the constant term of $E_{p q}$ at the cusp $1 / p$ is 0 . Similarly, the constant term of $E_{p q}$ at the cusp $1 / q$ is 0 . The constant term of $E_{p q}$ at the cusp $\infty$ is $\frac{1}{24}(p q-1)$ and at 0 is $\frac{1}{24}(1-p q) /(p q)$.

Using Lemmas 36 and 38, we have:

\section{Corollary 39.}

$$
(1-p q) e_{p q}=\sum_{x \in(\mathbb{Z} / p q \mathbb{Z})^{*}} F_{p q}(1, x)\left\{0, \frac{1}{x}\right\} .
$$


Remark 40. For the Eisenstein series $E_{p} \in E_{2}\left(\Gamma_{0}(p)\right), 1 / p$ represents the cusp $\infty$ and $1 / q$ represents the cusp 0 . We deduce that $a_{0}\left(E_{p}\left[\beta_{0}\right]\right)=\frac{1}{24}(p-1)$ and $a_{0}\left(E_{p}\left[\gamma_{0}\right]\right)=\frac{1}{24}(1-p) / p$. For the other Eisenstein series $E_{q} \in E_{2}\left(\Gamma_{0}(q)\right), 1 / q$ represents the cusp $\infty$ and $1 / p$ represents the cusp 0 . We deduce that $a_{0}\left(E_{q}\left[\gamma_{0}\right]\right)=$ $\frac{1}{24}(q-1)$ and $a_{0}\left(E_{q}\left[\beta_{0}\right]\right)=\frac{1}{24}(1-q) / q$.

\section{References}

[Agashe 2000] A. S. Agashe, The Birch and Swinnerton-Dyer formula for modular abelian varieties of analytic rank zero, thesis, University of California, Berkeley, 2000, available at http:// search.proquest.com/docview/304623798. MR 2701025

[Banerjee 2014] D. Banerjee, "A note on the Eisenstein elements of prime square level”, Proc. Amer. Math. Soc. 142:11 (2014), 3675-3686. MR 3251709 Zbl 06345376

[Calegari and Emerton 2005] F. Calegari and M. Emerton, "On the ramification of Hecke algebras at Eisenstein primes”, Invent. Math. 160:1 (2005), 97-144. MR 2007h:11066 Zbl 1145.11314

[Cremona 1997] J. E. Cremona, Algorithms for modular elliptic curves, 2nd ed., Cambridge University Press, 1997. MR 99e:11068 Zbl 0872.14041

[Diamond and Shurman 2005] F. Diamond and J. Shurman, A first course in modular forms, Graduate Texts in Mathematics 228, Springer, New York, 2005. MR 2006f:11045 Zbl 1062.11022

[Drinfeld 1973] V. G. Drinfeld, “Две теоремы о модулярных кривых”, Funkcional. Anal. $i$ Priložen. 7:2 (1973), 83-84. Translated as "Two theorems on modular curves" in Funct. Anal. Appl. 7:2 (1973), 155-156. MR 47 \#6705 Zbl 0285.14006

[Lang 1995] S. Lang, Introduction to modular forms, Grundlehren der Mathematischen Wissenschaften 222, Springer, Berlin, 1995. MR 96g:11037 Zbl 0344.10011

[Manin 1972] J. I. Manin, “Параболические точки и дзета-функции модулярных кривых”, Izv. Akad. Nauk SSSR Ser. Mat. 36:1 (1972), 19-66. Translated as "Parabolic points and zeta-functions of modular curves" in Math. USSR Izv. 6:1 (1972), 19-64. MR 47 \#3396 Zbl 0243.14008

[Mazur 1977] B. Mazur, "Modular curves and the Eisenstein ideal", Inst. Hautes Études Sci. Publ. Math. 47 (1977), 33-186. MR 80c:14015 Zbl 0394.14008

[Mazur 1979] B. Mazur, "On the arithmetic of special values of $L$ functions", Invent. Math. 55:3 (1979), 207-240. MR 82e:14033 Zbl 0426.14009

[Merel 1993] L. Merel, "Intersections sur des courbes modulaires", Manuscripta Math. 80:3 (1993), 283-289. MR 94k:14014 Zbl 0812.14013

[Merel 1995] L. Merel, "Homologie des courbes modulaires affines et paramétrisations modulaires", pp. 110-130 in Elliptic curves, modular forms, and Fermat's last theorem (Hong Kong, 1993), edited by J. Coates and S. T. Yau, Series in Number Theory 1, International Press, Cambridge, MA, 1995. MR 97a:11094 Zbl 0845.11023

[Merel 1996a] L. Merel, "Bornes pour la torsion des courbes elliptiques sur les corps de nombres", Invent. Math. 124:1-3 (1996), 437-449. MR 96i:11057 Zbl 0936.11037

[Merel 1996b] L. Merel, "L'accouplement de Weil entre le sous-groupe de Shimura et le sous-groupe cuspidal de $J_{0}(p)$ ", J. Reine Angew. Math. 477 (1996), 71-115. MR 97f:11045 Zbl 0859.11036

[Miranda 1995] R. Miranda, Algebraic curves and Riemann surfaces, Graduate Studies in Mathematics 5, American Mathematical Society, Providence, RI, 1995. MR 96f:14029 Zbl 0820.14022

[Miyake 1976] T. Miyake, Hokei keishiki to seisiiron, Kinokuniya, Tokyo, 1976. Translated by Yoshitaka Maeda as Modular forms, Springer, Berlin, 1989. Corrected 2nd printing in 2006. MR 2006g:11084 Zbl 1159.11014 
[Ogg 1974] A. P. Ogg, "Hyperelliptic modular curves", Bull. Soc. Math. France 102 (1974), 449-462. MR 51 \#514 Zbl 0314.10018

[Stevens 1982] G. Stevens, Arithmetic on modular curves, Progress in Mathematics 20, Birkhäuser, Boston, 1982. MR 87b:11050 Zbl 0529.10028

[Stevens 1985] G. Stevens, "The cuspidal group and special values of $L$-functions", Trans. Amer. Math. Soc. 291:2 (1985), 519-550. MR 87a:11056 Zbl 0579.10011

Received April 14, 2015. Revised June 25, 2015.

DEBARGHA BANERJEE

DEPARTMENT OF MATHEMATICS

Indian Institute of ScIENCE Education AND RESEARCh, PunE

PASHAN ROAD

PASHAN

PUNE 411008

INDIA

debargha@iiserpune.ac.in

SRILAKSHMI KRISHNAMOORTHY

DEPARTMENT OF MATHEMATICS

INDIAN INSTITUTE OF TECHNOLOGY MADRAS

SARDAR PATEL ROAD

CHENNAI

ADYAR 600036

INDIA

srilakshmi@iitm.ac.in 


\title{
PACIFIC JOURNAL OF MATHEMATICS
}

\author{
msp.org/pjm
}

Founded in 1951 by E. F. Beckenbach (1906-1982) and F. Wolf (1904-1989)

\section{EDITORS}

Don Blasius (Managing Editor)

Department of Mathematics

University of California

Los Angeles, CA 90095-1555

blasius@math.ucla.edu

\author{
Paul Balmer \\ Department of Mathematics \\ University of California \\ Los Angeles, CA 90095-1555 \\ balmer@math.ucla.edu \\ Robert Finn \\ Department of Mathematics \\ Stanford University \\ Stanford, CA 94305-2125 \\ finn@math.stanford.edu \\ Sorin Popa \\ Department of Mathematics \\ University of California \\ Los Angeles, CA 90095-1555 \\ popa@math.ucla.edu
}

\author{
Vyjayanthi Chari \\ Department of Mathematics \\ University of California \\ Riverside, CA 92521-0135 \\ chari@math.ucr.edu \\ Kefeng Liu \\ Department of Mathematics \\ University of California \\ Los Angeles, CA 90095-1555 \\ liu@math.ucla.edu \\ Jie Qing \\ Department of Mathematics \\ University of California \\ Santa Cruz, CA 95064 \\ qing@ cats.ucsc.edu
}

\section{PRODUCTION}

Silvio Levy, Scientific Editor, production@msp.org

\section{SUPPORTING INSTITUTIONS}

ACADEMIA SINICA, TAIPEI

CALIFORNIA INST. OF TECHNOLOGY

INST. DE MATEMÁTICA PURA E APLICADA

KEIO UNIVERSITY

MATH. SCIENCES RESEARCH INSTITUTE

NEW MEXICO STATE UNIV.

OREGON STATE UNIV.

\author{
STANFORD UNIVERSITY \\ UNIV. OF BRITISH COLUMBIA \\ UNIV. OF CALIFORNIA, BERKELEY \\ UNIV. OF CALIFORNIA, DAVIS \\ UNIV. OF CALIFORNIA, LOS ANGELES \\ UNIV. OF CALIFORNIA, RIVERSIDE \\ UNIV. OF CALIFORNIA, SAN DIEGO \\ UNIV. OF CALIF., SANTA BARBARA
}

\author{
Daryl Cooper \\ Department of Mathematics \\ University of California \\ Santa Barbara, CA 93106-3080 \\ cooper@math.ucsb.edu \\ Jiang-Hua Lu \\ Department of Mathematics \\ The University of Hong Kong \\ Pokfulam Rd., Hong Kong \\ jhlu@maths.hku.hk \\ Paul Yang \\ Department of Mathematics \\ Princeton University \\ Princeton NJ 08544-1000 \\ yang@math.princeton.edu
}

These supporting institutions contribute to the cost of publication of this Journal, but they are not owners or publishers and have no responsibility for its contents or policies.

See inside back cover or msp.org/pjm for submission instructions.

The subscription price for 2016 is US $\$ 440 /$ year for the electronic version, and $\$ 600 /$ year for print and electronic.

Subscriptions, requests for back issues and changes of subscribers address should be sent to Pacific Journal of Mathematics, P.O. Box 4163, Berkeley, CA 94704-0163, U.S.A. The Pacific Journal of Mathematics is indexed by Mathematical Reviews, Zentralblatt MATH, PASCAL CNRS Index, Referativnyi Zhurnal, Current Mathematical Publications and Web of Knowledge (Science Citation Index).

The Pacific Journal of Mathematics (ISSN 0030-8730) at the University of California, c/o Department of Mathematics, 798 Evans Hall \#3840, Berkeley, CA 94720-3840, is published twelve times a year. Periodical rate postage paid at Berkeley, CA 94704, and additional mailing offices. POSTMASTER: send address changes to Pacific Journal of Mathematics, P.O. Box 4163, Berkeley, CA 94704-0163.

PJM peer review and production are managed by EditFLOW ${ }^{\circledR}$ from Mathematical Sciences Publishers.

\section{PUBLISHED BY}

\section{mathematical sciences publishers \\ nonprofit scientific publishing}

http://msp.org/

(C) 2016 Mathematical Sciences Publishers 


\title{
PACIFIC JOURNAL OF MATHEMATICS
}

\author{
Volume $281 \quad$ No. $2 \quad$ April 2016
}

The Eisenstein elements of modular symbols for level product of two 257 distinct odd primes

DEBARGHA BANERJEE and SRILAKSHMI KRISHNAMOORTHY

Primitively generated Hall algebras

ARKADY BERENSTEIN and JACOB GREENSTEIN

Generalized splines on arbitrary graphs

Simcha Gilbert, JULiAnNA TyMOCZKO and SHIRA ViEL

Good traces for not necessarily simple dimension groups

DAVID HANDELMAN

On Fourier coefficients of certain residual representations of symplectic groups

DIHUA JIANG and BAIYING LIU

On the existence of central fans of capillary surfaces

AMMAR KHANFER

Surfaces of prescribed mean curvature $H(x, y, z)$ with one-to-one central projection onto a plane

FRIEDRICH SAUVIGNY 